\title{
Standardized Verification of Fuel Cycle Modeling
}

\author{
${ }^{1}$ B. Feng, ${ }^{2}$ B. Dixon, ${ }^{3}$ E. Sunny, ${ }^{4}$ A. Cuadra, ${ }^{2}$ J. Jacobson, ${ }^{4,3}$ N. R. Brown, ${ }^{3}$ J. Powers, ${ }^{3}$ A. Worrall, ${ }^{1}$ S. \\ Passerini, and ${ }^{5} \mathrm{R}$. Gregg \\ ${ }^{1}$ Argonne National Laboratory \\ ${ }^{2}$ Idaho National Laboratory \\ ${ }^{3}$ Oak Ridge National Laboratory \\ ${ }^{4}$ Brookhaven National Laboratory \\ ${ }^{5}$ UK National Nuclear Laboratory
}

\begin{abstract}
A nuclear fuel cycle systems modeling and code-to-code comparison effort was coordinated across multiple national laboratories to verify the tools needed to perform fuel cycle analyses of the transition from a once-through nuclear fuel cycle to a sustainable potential future fuel cycle. The high-level objective was to derive information that can be used by the US Department of Energy (DOE) to help inform future research and development decisions. For this verification study, a simplified transition scenario was developed to serve as a test case for the four systems codes involved (DYMOND, VISION, ORION, and MARKAL), each used by a different laboratory participant. In addition, all participants produced spreadsheet solutions for the test case to check all the mass flows and reactor/facility profiles on a year-by-year basis throughout the simulation period. The test case specifications describe a transition from the current US fleet of light water reactors to a future fleet of sodium-cooled fast reactors that continuously recycle transuranic elements as fuel. After several initial coordinated modeling and calculation attempts, it was revealed that most of the differences in code results were not due to different code algorithms or calculation approaches, but due to different interpretations of the input specifications among the analysts. Therefore, the specifications for the test case itself were iteratively updated to remove ambiguity and to help calibrate interpretations. In addition, a few corrections and modifications were made to the codes as well, which led to excellent agreement between all codes and spreadsheets for this test case. Although no fuel cycle transition analysis codes matched the spreadsheet results exactly, all remaining differences in the results were due to fundamental differences in code structure and/or were thoroughly explained. The specifications and example results are provided so that they can be used to verify additional codes in the future for such fuel cycle transition scenarios.
\end{abstract}

\section{Introduction}

Numerous nuclear fuel cycle system code benchmarks and comparisons have been performed over the last decade, coinciding with the rapid development of new codes due to improvements in computational capabilities, new software platforms, and the need for various institutions to provide technical feedback on potential fuel cycle strategies and policies. Many of these studies [MIT 2009, EC-PUMA 2010, NEA 2012] were performed within the framework of established international organizations (EC-PUMA, NEA) or organized as an ad hoc study with voluntary contributions from participants (MIT). These studies achieved different levels of agreement depending on the scenarios analyzed and values compared, varying from excellent agreement in annual mass flows and inventories to general agreement in terms of trends. Since validation is challenging for these types of codes, these comparison studies helped develop confidence in the results from these forecasting codes. In addition, many of these codes were developed independently with limited feedback due to the lack of a widely-established user base. Therefore, such studies are also great opportunities for the developers and users to calibrate interpretations as well as modify/debug the codes themselves. 
The work presented in this paper is a code verification study similar to previous studies but unique in several ways: 1) all participants were working under a common project funded and coordinated by the US Department of Energy (DOE), 2) a strict level of agreement between codes was targeted, and 3) during the verification process, modifications were made to the participating codes in the pursuit of achieving such a high level of agreement. The codes involved in this verification study are DYMOND [Yacout $e t$ al., 2005], VISION [Jacobson et al., 2011], ORION [Gregg and Grove, 2012], and MARKAL [Shay et al., 2006]. Many authors of this paper were also participants in these previous activities; consequently, many of the lessons learned from preceding experiences were applied toward this new collaborative effort.

\subsection{Context of Work}

The present code verification study was conducted in a coordinated effort as part of the Fuel Cycle Options (FCO) Campaign, a multi-laboratory collaborative effort within the US DOE-Nuclear Energy's Office of Fuel Cycle Technologies. The Campaign recently completed a multi-year evaluation and screening of a comprehensive set of nuclear fuel cycle options [Wigeland et al., 2014], assessing the potential for performance improvements compared to the existing once-through cycle in the US across a wide range of criteria with an objective of informing on research and development priorities. It was found that regardless of the relative importance of the benefit criteria, the fuel cycles that consistently scored high overall performance were ones that involved continuous recycling of co-extracted U/Pu or U/TRU in fast spectrum critical reactors. A key feature of the evaluation and screening study was that the performance for each fuel cycle was evaluated assuming a fully-deployed nuclear energy system at equilibrium to properly understand the end-state benefits. Based on the findings from this study, the next step in understanding research and development needs is to assess and inform on the fuel cycle transitions from the current state to these promising future end-states. However, before initiating the time-dependent transition analyses, it was necessary to assess the systems analysis tools and capabilities within the Campaign to 1) ensure that the fuel cycle simulations produce consistent results, 2) identify reasons for differences between code behaviors and analyst interpretations, and 3) identify limitations or advantages of individual codes.

\subsection{Objectives}

The main objectives of this paper are: 1) to show the consistent agreement among the participating fuel cycle codes for an artificial test scenario involving transition, thereby demonstrating confidence in the code calculations and modeling capabilities, 2) to show the level of effort and iteration required to obtain agreement between codes, and 3 ) to publish the scenario specifications and results so that other fuel cycle codes can be used to repeat this verification exercise.

First, each of the three fuel cycle codes (DYMOND, ORION, and VISION) and one market analysis code (MARKAL) involved in this verification study will be briefly described. Then, the specifications and methodology for the test transition scenario for this verification exercise will be discussed. As briefly mentioned, part of this verification exercise also involved using external calculations, i.e., spreadsheets, to serve as an additional means of cross-checking. To avoid biasing the presentation of the results toward a particular code solution, the spreadsheet results are first presented in this paper and then a few selected results from each code will be presented to highlight any differences from the spreadsheet solution as well as differences among each of the code results. 


\section{Code Descriptions}

Detailed descriptions of system dynamics and fuel cycle systems modeling are not provided in this paper since it is assumed that the reader has a general understanding of these concepts. Only brief descriptions are provided in this section, emphasizing some of the differences that will be pertinent to the results. Additional information on these codes is provided in their respective literature listed in the references. Table 1 summarizes the laboratories that have completed this benchmark and the tools they used (each also produced the same spreadsheet solution).

Table 1 Participants and codes/programs used for fuel cycle modeling verification effort

\begin{tabular}{|c|c|c|}
\hline Participant & Code/Tool Used & Code/Tool Type \\
\hline Argonne National Laboratory (ANL) & DYMOND & Nuclear fuel cycle \\
Idaho National Laboratory (INL) & VISION & Nuclear fuel cycle \\
Oak Ridge National Laboratory (ORNL) & ORION & Nuclear fuel cycle \\
Brookhaven National Laboratory (BNL) & MARKAL & Energy market \\
\hline
\end{tabular}

\subsection{DYMOND Description}

DYMOND [Yacout et al., 2005], the main software tool used at ANL for this study, is a nuclear fuel cycle system dynamics model run within the iThink software with Microsoft Excel templates for data input/output. The code was first developed in 2001 at ANL for the Gen IV Fuel Cycle Crosscut Group activities and is a predecessor to several system dynamics codes in wide use today. Since 2012, the code structure and user interface were heavily updated while maintaining its relative simplicity. Like all codes involved in this study, many corrections and updates were made as a result of this study as well as other verification studies that are currently underway.

The major inputs to the code are the reactor and fuel characteristics, the fuel cycle facility properties, the various pathways of each fuel type (enrichment, recycling, storage, etc.), and the nuclear power demand as a function of time. The code uses externally-calculated fuel cycle recipes for both the input and output fuel compositions. Currently only described in lumped materials (U, Pu, etc.), The major output is the reactor fleet composition (installed capacities of each type of reactor) over time. All other system data including mass flows (mining rates, fabrication rates, reprocessing rates, etc.) and inventories (spent fuel inventory, recovered uranium, etc.) can be output via an Excel spreadsheet based on the user's selection. Typical run times are less than a minute for scenarios lasting over a century with month-long time steps. The time steps can be varied at the user's discretion.

During the development of the DYMOND code, the ability for the user to easily understand the code's behavior and variables was considered a priority over model sophistication and hidden logic and automation. This allows a higher level of manual control and input flexibility that allows a scenario to fail. For example, when there are insufficient fissile materials for fuel fabrication, the correct amount of existing reactor capacities will go into "idle" mode and the user-specified energy demand will not be met. In addition, a dynamic resource allocation module was implemented to allow prioritization of fissile material allocation to different reactor technologies based on user-specified priorities. One of the original simplifications that has been maintained is that individual isotopes are not tracked; materials are lumped into categories such as fission product (FP), minor actinide (MA), and several types of $\mathrm{U}$ and $\mathrm{Pu}$. In addition, radioactive decay is not currently implemented. These approximations were acceptable for the present verification study since only lumped elements were modeled to allow for simple spreadsheet comparisons. Any reactor and fuel types can be modeled so long as the technical details are provided as input, and individual reactors can be simulated to transition from one fuel type to a completely new one within a few cycles. 


\subsection{VISION Description}

VISION [Jacobson et al., 2010] was developed by the US DOE Advanced Fuel Cycle Initiative (AFCI) program (predecessor to the FCO campaign) as an evolution of the DYMOND code. It was developed to provide modeling capabilities that exceeded the limits at the time of DYMOND's software platform. VISION is hence an extension of DYMOND that was re-structured and moved to the PowerSim software platform along with Excel worksheets used for input and output data. Development of the code has since diverged from that of DYMOND to the point where comparison studies are expected to reveal differences in the results.

The initial version of VISION was US-centric and was used as the campaign's primary scenario analysis tool for several years [Dixon et al., 2010]. A major revision of the code was undertaken at around 2010 to generalize the code, adding flexibility to model any type of reactor with up to 10 reactor fleets, 40 separation matrices, and a number of other capabilities. As part of this revision, a series test cases and benchmark results was developed to support verification of basic functionality and ongoing confirmation that changes within the code and/or its supporting files do not impair the results. These efforts, which include the present verification study with other codes, fit within an overall software development framework which manages the change processes and helps ensure quality and version control.

Like DYMOND, VISION simulates the nuclear fuel cycle not based on individual facilities but at the system level, i.e., a fleet of nuclear reactors with associated front-end (fuel resources and fabrication processes) and back-end (storage and disposal) facilities. While facilities are individually constructed and retired, they are primarily represented as changes to system fleet capacities. The code models the dynamic transition from an initial state to an end state and provides resource needs, mass flows, compositions, facility needs, and other time-dependent fuel cycle data to help explore the impacts of various scenarios, transition strategies, and policy decisions. The architecture of the code is modular and therefore adaptable and extensible for addressing other needs such as exploring nuclear waste issues, economic aspects, and non-proliferation considerations.

VISION is capable of simulating a nuclear fuel cycle composed of multiple reactor types, each using user-specified fuel types and reprocessing strategies. A great deal of flexibility is provided for the user to specify both the material routing between fuel cycle facilities and the material transformations that occur within facilities and to vary these as needed to explore the evolution of fuel and reprocessing strategies during the transition. In addition, individual isotopes and radioactive decay can be modeled along with up to 5 different fuel compositions for a single type of fuel to more accurately reflect the isotopic evolution of fuel through multiple passes through a reactor.

\subsection{ORION Description}

ORION [Gregg and Grove, 2012] was used by ORNL to take part in this verification exercise and to perform fuel cycle modeling for transition analysis activities. ORION is a fuel cycle modeling code (written in $\mathrm{C}++$ ) that has been developed for more than 15 years by the UK National Nuclear Laboratory (NNL) and can be used on a Windows, Linux, or Apple computer. This code was chosen by ORNL because it is independent from the other tools used within the Campaign and because it provided some unique features that may support modeling needs, such as high-fidelity radioactive decay for over 2000 nuclides based on source nuclear library data and use of cross sections for depletion rather than material recipes. Furthermore, the graphical user interface is relatively easy and intuitive to use, allowing the user to construct a visual representation of the fuel cycle with run times on the order of seconds to a few minutes. ORION can model various nuclear facilities as individual objects. Several of these objects can be grouped together to represent many small-scale facilities that support one reactor, or one object can represent a large-scale facility that represents the behavior of a fleet. Therefore, the objects in ORION can be used to represent individual facilities or a fleet of facilities. The Reactor Dynamic Control tool in ORION can be used to automate the commissioning and decommissioning of selected reactors based on 
the electricity demand and decommissioning or commissioning profiles of other reactors. The "change order" feature allows for the prioritization of facilities, allowing mass flows through various streams to be prioritized over others. ORION also allows the user to specify individual reactor lifetimes for various years in addition to the "year vs. mass demand" and "year vs. energy demand" features that were available in previous versions.

The program can track over 2500 nuclides (most of which are short-lived) and can model both decay and in-reactor irradiation. Prior to an ORION analysis, detailed lattice physics calculations are completed using external codes to generate either burnup-dependent cross section libraries or fuel recipes for each reactor and fuel type. When using burnup-dependent cross sections, ORION can model in-reactor radionuclide inventory changes and can estimate any feedback effects on new fuel compositions.

ORION is able to explicitly model driver and blanket fuel streams, and the isotopic evolution with burnup in those streams, separately for multi-region systems. This enables changes in fuel, core, and reactor designs, including breeding ratios and mass demands for each component of the fuel supply, to be explicitly tracked. ORION has the functionality to scale the blanket region (or others) with a parent reactor to ensure fuel demands scale appropriately.

ORION can simulate the full range of nuclear-related facilities, as well as all reactor types (e.g., LWRs, FRs, MSRs, etc.) and fuel types (e.g., U, Pu, Th, MA, etc.). It is also capable of modeling detailed individual reactors or entire fleets. The user also has the flexibility to choose material flows, separations and stream priorities.

\subsection{MARKAL Description}

BNL is employing MARKAL [Shay et al., 2006] to help understand and evaluate external factors that affect fuel cycle transition. MARKAL was developed as an energy system and associated resource flow optimization tool built using linear and mixed-integer linear programming techniques. MARKAL is heavily data-driven and the relevance of the simulation results depends on the quality of the data. The end-user generates a comprehensive mapping, including structure and technologies of the energy system. The overall energy system includes energy sources, energy carriers, energy conversion, and energy delivery. An associated database is required to model the technologies and resources within energy systems, including: technology availability and performance, inputs and outputs for materials, fixed and variable costs, and pollutant emissions. Based on the models, structures, constraints, and data, MARKAL calculates the least cost set of technologies over time within the bounds of the user-defined constraints. Key output data from the model include a determination of the technological mix at intervals into the future, estimates of total system cost, energy demand (by type and quantity), emissions, and energy commodity prices. Thus, MARKAL provides the capability to integrate other models of energy demand and competing energy sources.

Although MARKAL was not developed specifically as a nuclear fuel cycle analysis tool like DYMOND, VISION, and ORION, it was modified and adapted for use in this verification study as a way of demonstrating its flexible and expansive applications. One of the potential advantages of using MARKAL for transition analysis is the capability to integrate other complex models of energy demand and competing non-nuclear energy sources during the later phases of the Campaign's transition analysis effort. Additional details of the modeling are provided in the results section. 


\section{Methodology}

The general approach for verification studies of this nature is to first develop and issue the specifications to all participants and then each would independently produce the results and compare them at the end. This approach is straightforward but often leads to differences attributed to different interpretations of the specifications among the participants, especially if there is any ambiguity in the language. Therefore, the approach used in this study was as follows:

1) Develop first draft of the specifications for the test transition scenario;

2) Using both spreadsheets and codes, model the scenario and calculate the requested parameters for each year of the simulation;

3) Find all the differences in results between all spreadsheets and codes;

4) All differences must then be resolved or explained:

a) If the differences were due to different interpretations, then a common interpretation must be set with the specifications updated with more specific language;

b) If they were due to code errors then the codes would have to be corrected/modified;

c) Any remaining unresolved differences due to rounding and/or different code structures and algorithms must be explained.

5) After this iterative process, the final results for the test transition scenario were compared and presented in this paper.

Figure 1 shows a schematic summarizing the iterative development of the specifications and generation of the results. This paper does not go into all the details of this entire process but shows a few examples of the standardization and corrections that indicate the level of effort undertaken and demonstrate the necessity of using multiple codes for quality control.

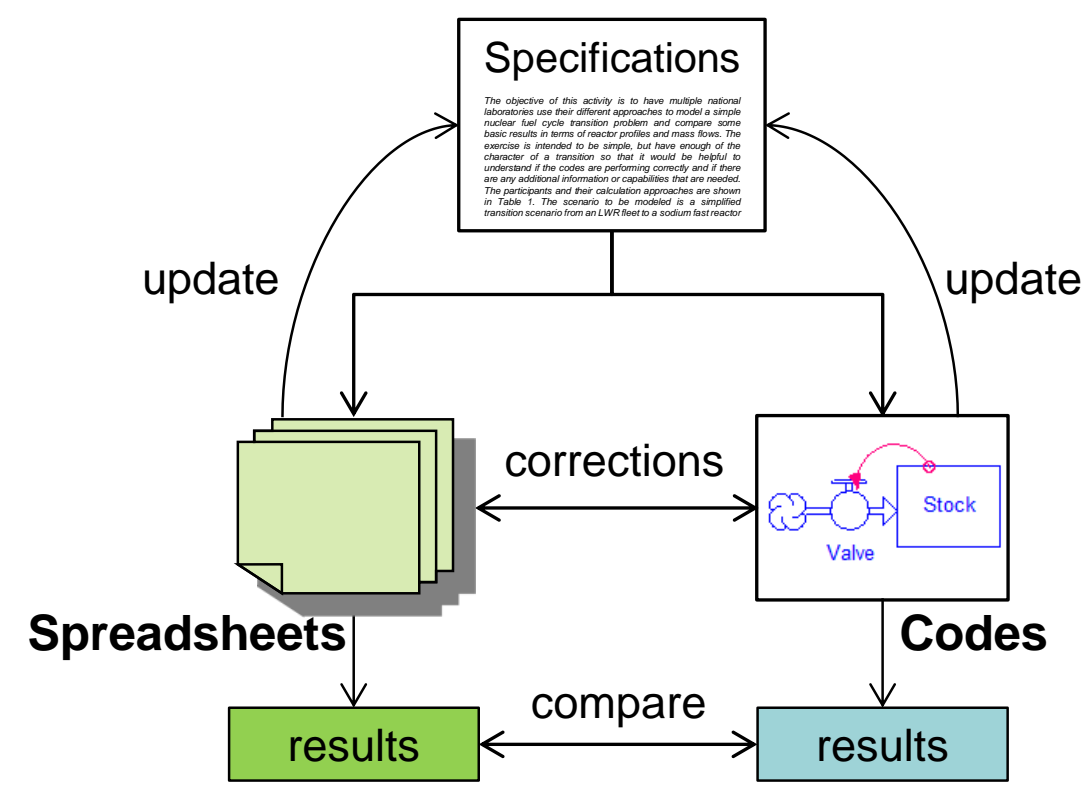

Figure 1 Iterative development of specifications and results for verification study 
The results required very precise definitions and a single interpretation of the specification language across all participants. For example, the first version of the specifications asked for "annual values" to be reported for mass flows but this could be interpreted in many ways as it does not specify whether they are instantaneous or cumulative, reported at the beginning or end of year, or reported as the minimum, mean, or maximum value. Therefore, for the successive versions of the specifications, each annual value that is to be calculated and compared was then affixed with a "value type" to remove such ambiguity. The first runs also revealed different interpretations for when each instantaneous process occurs and when the values are reported during the year. The difficulty of explaining the exact timing and sequence of events required adopting figures to remove all ambiguity in the specifications, as shown in the scenario specifications in the next section.

In addition to the specifications, a standardized output template in Excel was also iteratively developed as part of this verification study to have all results plotted on the same figures. This enabled the results from the different codes to be plotted using the same scales, color coding, units, and value types for ease of comparison and interpretation. The raw results from each code were either directly exported or transferred via scripting to the Excel standardized output template which enabled ease of comparison and producing many results in a short amount of time.

Although the vast majority of differences between the codes were due to interpretive differences, there were a few code corrections or modifications that had to be made through the cross-checking process with the spreadsheet calculations. For example, a few timing offsets (even just by one or two partial-year time steps) in some of the codes were revealed when compared with the spreadsheet, which only calculated values based on whole-year time steps. In return, some of the codes revealed common modeling errors in several spreadsheets produced by different analysts that would otherwise not have been found. At times, this correction process required tracking the smallest unit of mass throughout each code to fully understand the calculations and not treat the codes like "black boxes". The specifications described in the next section are the final versions after multiple updates via this iterative process and can be used to repeat this verification exercise with other codes. 


\section{Scenario Specifications}

The test scenario for this verification study is a simplified transition from a fleet of one hundred 1000MWe light-water reactors (LWRs) to a fleet of three hundred 333.3-MWe sodium fast reactors (SFRs), under a scenario in which there is $0 \%$ growth in nuclear energy demand. It was designed to be simple so that spreadsheets or other forms of external calculations could be used to calculate the mass flows, but also exhibit the features of a transition from one reactor technology to another. It should be noted that this scenario is only an example for testing purposes and may not be representative of a realistic fuel cycle transition scenario. However, the simplicity of the assumptions allowed for easier benchmarking and the scenario served the purpose of calibrating the tools used for fuel cycle analysis.

\subsection{General Parameters}

The general parameters of the scenario are shown in Table 2. The scenario starts in the beginning of the year 2015 with 100 LWRs, each at 1 GWe capacity, totaling 100 GWe of installed LWR capacity. The simulation period is 100 years to allow enough time for the complete transition of the existing LWR fleet into the fleet of SFRs. The capacity factor is 0.90 for both reactor types and the nuclear power demand remains a constant at $90 \mathrm{GWe}$. For simplicity and easier accounting of mass flows, no material process losses (fabrication, separation, enrichment, conversion, etc.) were modeled. Also, it was assumed that no existing materials in 2015 other than natural uranium are available for use in fuel fabrication. This implies that no depleted uranium, separated Pu, LWR used nuclear fuel (UNF), etc. are available at the beginning of the scenario. However, any of these materials produced by the reactor fleet or fuel cycle processes during the simulation period can be used. For example, LWR UNF discharged in 2015 and after can be recycled to make fast reactor fuel.

Table 2 General parameters of test transition scenario

\begin{tabular}{|l|c|}
\hline \multicolumn{2}{|c|}{ General Parameters } \\
\hline Simulation Start Year & 2015 \\
Simulation End Year & 2115 \\
Initial Nuclear Capacity, GWe & 100 \\
Initial Electricity Demand, GWe-y & 90 \\
Electricity Demand Growth Rate, \%/y & 0 \\
${ }^{235}$ U in Natural Uranium, wt\% & 0.711 \\
${ }^{235}$ U in Depleted Uranium, wt\% & 0.25 \\
Natural Uranium Inventory, $\mathrm{U}$ & unlimited \\
\hline
\end{tabular}




\subsection{Reprocessing}

As shown in Table 3, commercial reprocessing of LWR UNF begins in 2025 with a fixed maximum capacity of 2,000 tons of initial heavy metal (tIHM) of UNF per year. When referring to UNF, tIHM includes heavy metals (HM) and fission products but no fuel matrix materials, such as oxygen or zirconium. SFR UNF reprocessing is available immediately after the SFR UNF is cooled and the capacity is unlimited.

Table 3 Reprocessing plant parameters of test transition scenario

\begin{tabular}{|l|c|}
\hline \multicolumn{2}{|c|}{ Reprocessing Plants } \\
\hline LWR UNF Reprocessing Start Year & 2025 \\
LWR UNF Reprocessing Capacity, tIHM/y & 2000 \\
SFR UNF Reprocessing Start Year & when needed \\
SFR UNF Reprocessing Capacity, tIHM/y & as needed \\
\hline
\end{tabular}

\subsection{Existing LWR Retirement Profile}

The main feature in this test scenario is that for each of the 100 existing LWRs retired, 3 SFRs are constructed at the same time to maintain a constant level of energy production throughout the simulation. For this test scenario, the LWR retirement profile (and effectively, the SFR deployment profile) is provided as input for all codes and spreadsheets to have the same operating reactors at all times, thereby removing a large source of discrepancy that has been found in previous benchmarks due to the different construction and retirement algorithms of each code.

The specified LWR retirement profile, as shown in Table 4, was calculated with spreadsheets based on the rule that as soon as sufficient fuel material is available to start-up and maintain 1 GWe of SFR reactor production, 3 SFRs (totaling $1 \mathrm{GWe}$ ) are constructed and $1 \mathrm{LWR}$ is retired. A key constraint that led to this profile was to minimize the unused TRU inventory in terms of the point values (the annual surplus that is not needed after all the requested TRU is sent to fuel fabrication). This is different from typical fuel cycle scenarios in which existing LWRs are only retired when they reach the end of their assumed operational lifetimes.

It must be emphasized that each reactor start-up or retirement occurs at the beginning of each year during the first time step. It was decided that for this study, all facility state-change events (start-ups and retirements) in a year occur simultaneously, followed by all production and material flows. Inventories and capacities are reported at the completion of the state-change events and production and material flows. Given this convention, in the first year of operation a facility generates an energy product (electricity, separated materials, etc.), but no product is produced in the year a facility is retired. For example, Figure 2 shows the refueling scheme and period of electricity generation for a reactor with a 60year lifetime (online in 2026 and offline in 2086). 
Table $4 \mathrm{LWR}$ retirement profile for test transition scenario

\begin{tabular}{|c|c|c|c|c|c|}
\hline Year & Total LWRs Installed & $\begin{array}{c}\text { LWR } \\
\text { Retirements }\end{array}$ & Year & Total LWRs Installed & $\begin{array}{c}\text { LWR } \\
\text { Retirements }\end{array}$ \\
\hline 2015 & 100 & 0 & 2056 & 17 & 0 \\
\hline 2016 & 100 & 0 & 2057 & 16 & 1 \\
\hline 2017 & 100 & 0 & 2058 & 14 & 2 \\
\hline 2018 & 100 & 0 & 2059 & 14 & 0 \\
\hline 2019 & 100 & 0 & 2060 & 13 & 1 \\
\hline 2020 & 100 & 0 & 2061 & 12 & 1 \\
\hline 2021 & 100 & 0 & 2062 & 11 & 1 \\
\hline 2022 & 100 & 0 & 2063 & 11 & 0 \\
\hline 2023 & 100 & 0 & 2064 & 10 & 1 \\
\hline 2024 & 100 & 0 & 2065 & 9 & 1 \\
\hline 2025 & 100 & 0 & 2066 & 9 & 0 \\
\hline 2026 & 97 & 3 & 2067 & 8 & 1 \\
\hline 2027 & 93 & 4 & 2068 & 8 & 0 \\
\hline 2028 & 91 & 2 & 2069 & 7 & 1 \\
\hline 2029 & 89 & 2 & 2070 & 7 & 0 \\
\hline 2030 & 86 & 3 & 2071 & 6 & 1 \\
\hline 2031 & 83 & 3 & 2072 & 6 & 0 \\
\hline 2032 & 80 & 3 & 2073 & 5 & 1 \\
\hline 2033 & 78 & 2 & 2074 & 5 & 0 \\
\hline 2034 & 75 & 3 & 2075 & 5 & 0 \\
\hline 2035 & 72 & 3 & 2076 & 4 & 1 \\
\hline 2036 & 70 & 2 & 2077 & 4 & 0 \\
\hline 2037 & 67 & 3 & 2078 & 4 & 0 \\
\hline 2038 & 64 & 3 & 2079 & 4 & 0 \\
\hline 2039 & 61 & 3 & 2080 & 4 & 0 \\
\hline 2040 & 59 & 2 & 2081 & 4 & 0 \\
\hline 2041 & 56 & 3 & 2082 & 4 & 0 \\
\hline 2042 & 53 & 3 & 2083 & 4 & 0 \\
\hline 2043 & 51 & 2 & 2084 & 4 & 0 \\
\hline 2044 & 48 & 3 & 2085 & 4 & 0 \\
\hline 2045 & 45 & 3 & 2086 & 4 & 0 \\
\hline 2046 & 42 & 3 & 2087 & 4 & 0 \\
\hline 2047 & 39 & 3 & 2088 & 3 & 1 \\
\hline 2048 & 37 & 2 & 2089 & 2 & 1 \\
\hline 2049 & 34 & 3 & 2090 & 2 & 0 \\
\hline 2050 & 31 & 3 & 2091 & 2 & 0 \\
\hline 2051 & 28 & 3 & 2092 & 2 & 0 \\
\hline 2052 & 26 & 2 & 2093 & 1 & 1 \\
\hline 2053 & 23 & 3 & 2094 & 1 & 0 \\
\hline 2054 & 20 & 3 & 2095 & 1 & 0 \\
\hline 2055 & 17 & 3 & 2096 & 0 & 1 \\
\hline
\end{tabular}




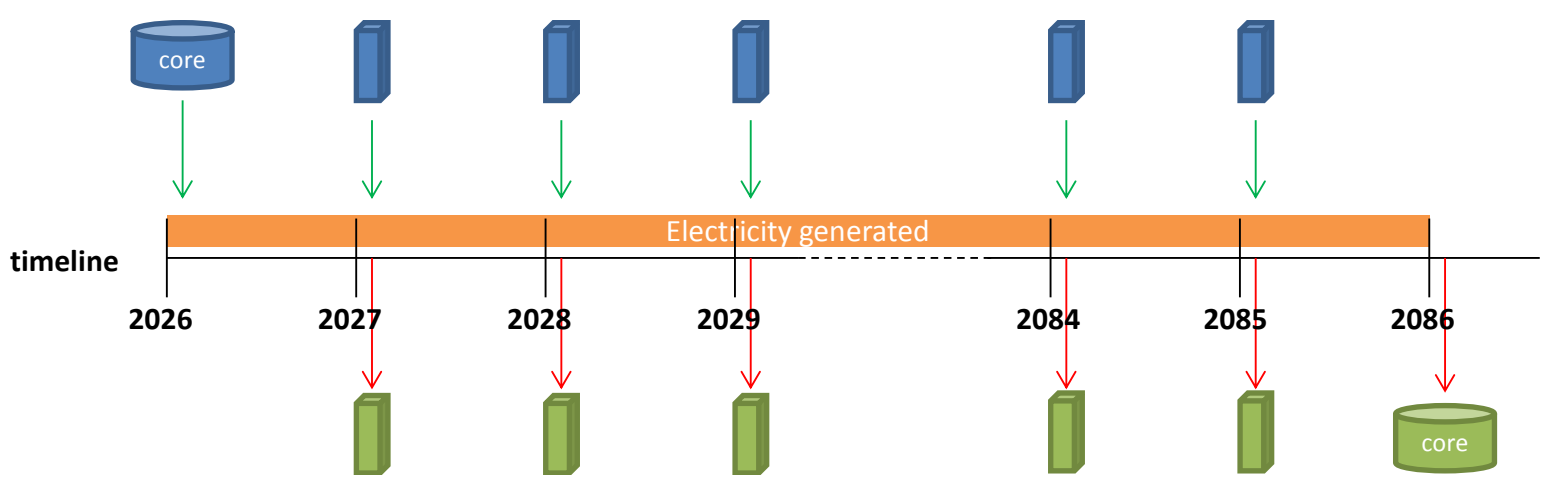

Figure 2 Example refueling scheme for a reactor with a 60-year lifetime

\subsection{Reactor and Fuel Properties}

Table 5 shows reactor properties for the LWR and SFR. Each SFR constructed is replaced after its 60year assumed operational lifetime with another new SFR. However, the assumed operational lifetimes of the LWRs are not specified because Table 4 already provides the LWR fleet's retirement profile. Note that both reactors have partial batches and 1 calendar year cycle lengths. This was specified to accommodate most system dynamics codes that model refueling as a continuous process in which small amounts of fuel are loaded and discharged during each time step. However, in this study, it was assumed that during the first cycle (first calendar year) there is no continuous refueling so some of the codes required modifications to model this behavior. ORION required modeling discrete numbers of batches, so the number of batches was rounded to a whole number and the cycle length was simply adjusted such that the product of the two values is constant. This will maintain the annual refueling load and core size, which can be calculated directly based on the combination of power, discharge burnup, batches, and cycle length:

$$
\text { Refueling Load }=\frac{\text { Thermal Power } \times \text { Capacity Factor } \times 365 \text { days }}{\text { Discharge Burnup }}
$$

Core Size $=$ Refueling Load $\times$ Batches $\times$ Cycle Length

Table 5 Reactor properties for test transition scenario

\begin{tabular}{|l|c|c|}
\hline \multicolumn{3}{|c|}{ Reactor Properties } \\
\hline & LWR & SFR \\
\hline Reactor Lifetime, y & N/A & 60 \\
Power, MWe & 1000 & 333.3 \\
Thermal Efficiency & 0.33 & 0.38 \\
Capacity Factor & 0.90 & 0.90 \\
Discharge Burnup, MWd/kgIHM & 50 & 73 \\
Batches & 4.5 & 3.96 \\
Cycle Length, calendar years & 1 & 1 \\
Refueling Load, tHM/y & 19.91 & 3.95 \\
Core Size, tHM & 89.59 & 15.63 \\
\hline
\end{tabular}


Table 6 shows the corresponding fuel compositions before loading and after discharge and cooling. For this scenario, all transuranic elements (TRU) are recovered from LWR and SFR UNF and recycled as the fissile material in new SFR fuel without any losses. There are no detailed isotopics for this comparison; the fuel compositions are defined only as lumped materials (U, TRU, and FP) even though it is known that TRU isotopic vectors from the discharged LWR and SFR fuels are different and may not match exactly the TRU isotopic vector of the SFR fresh fuel. This approximation was made for simplicity and easier material tracking and only requires that the total TRU mass be conserved during the processes associated with recycling. In addition, no decay was modeled except during irradiation so the fuel compositions at discharge and after the specified cooling period are the same. There is only 1 fresh fuel composition for each reactor type so there is no distinction between start-up core composition and equilibrium core composition. Since the fuel compositions in Table 6 are for equilibrium fuel, the start-up core's TRU requirements may be slightly overestimated for the first few cycles. Similarly, when a reactor is retired, its entire core is discharged, cooled, and recycled. These discharged cores were approximated to have the same compositions as used fuel discharged during equilibrium. The TRU inventory ratio (discharge divided by charge) is 1.012 for the SFR modeled.

Table 7 shows the time delays for each fuel cycle process and the order in which the TRU from different sources are used for fuel fabrication. Reprocessing was assumed to occur instantaneously, which is easy for the spreadsheets to model because they use 1-year time steps with the full year's worth of reprocessing throughput modeled as occurring at the beginning of the year. However, for some of the codes that used smaller time steps for each process, the total annual reprocessing throughput was split evenly across each time step within the year so only a fraction of the recovered materials was available at the beginning of each year. As soon as the UNF was cooled, it would be sent to reprocessing whether or not TRU was needed for fueling the SFRs. Figure 3 shows the time sequence of events for fuel mass flows corresponding to the time delays for LWR fuel in this scenario. When the fleet of SFRs has the choice of using TRU from SFR UNF or LWR UNF, it will first use up all the TRU extracted from SFR UNF, and then start using the TRU from LWR UNF. The same resource prioritization applies for the recovered uranium (RU) that is used as the fertile makeup. For this scenario, no depleted uranium or natural uranium is required since the RU inventory increases over time.

Table 6 Fuel compositions for test transition scenario

\begin{tabular}{|l|c|c|c|c|}
\hline & \multicolumn{2}{|c|}{ LWR Fuel Compositions, wt\% } & \multicolumn{2}{c|}{ SFR Fuel Compositions, wt \% } \\
\hline & Fresh & $\begin{array}{c}\text { At Discharge and } \\
\text { after Cooling }\end{array}$ & Fresh & $\begin{array}{c}\text { At Discharge and } \\
\text { after Cooling }\end{array}$ \\
\hline U & $100.0\left(4.3 \%{ }^{235} \mathrm{U}\right)$ & 93.44 & 86.13 & 78.30 \\
TRU & & 1.30 & 13.87 & 14.04 \\
FP & & 5.26 & - & 7.66 \\
\hline
\end{tabular}

Table 7 Fuel cycle time delays and material priorities of test transition scenario

\begin{tabular}{|l|c|c|}
\hline \multicolumn{2}{|c|}{ Fuel Properties } \\
\hline Fuel Type & LWR & SFR \\
Enrichment Time, y & UOX & (U,TRU)Zr \\
Fuel Fabrication Time, y & 1 & - \\
Reprocessing Time, y & 1 & 1 \\
UNF Cooling Time, y & 0 & 0 \\
UNF Storage Time, y & 4 & 1 \\
\hline
\end{tabular}




\begin{tabular}{|l|c|c|} 
Separated HM Storage Time, y & until used in fabrication & until used in fabrication \\
Fissile Material Source \#1 & LEU & SFR TRU \\
Fissile Material Source \#2 & - & LWR TRU \\
Fertile Material Source \#1 & - & SFR RU \\
Fertile Material Source \#2 & - & LWR RU \\
\hline
\end{tabular}

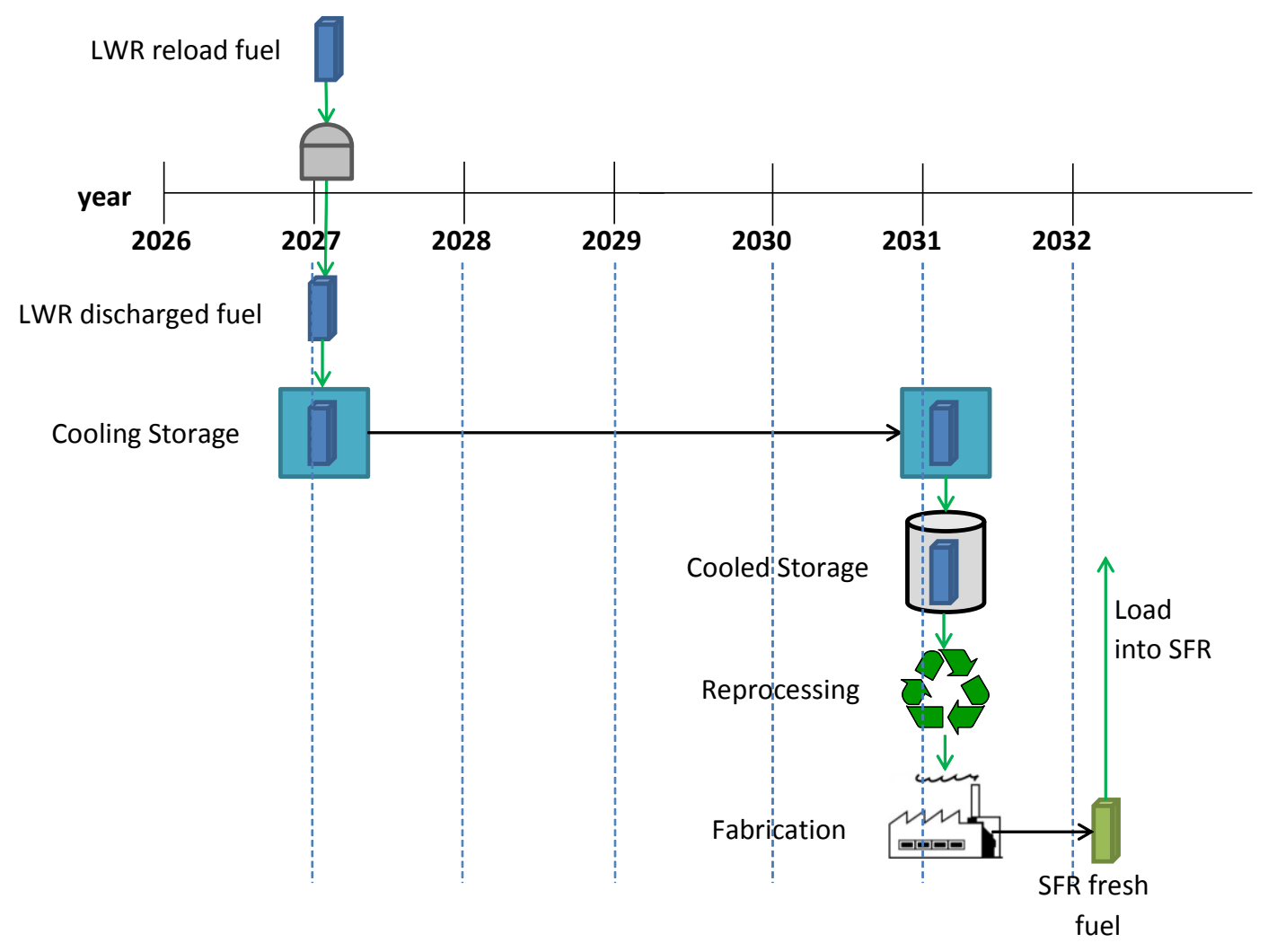

Figure 3 Time sequence of events for LWR fuel mass flow 


\section{Results}

For this verification exercise the same set of results ( 7 figures) were produced by each code and spreadsheet solution:

- LWR and SFR deployed capacity (GWe);

- LWRs retired and SFRs started up each year;

- LWR and SFR fresh fuel loading rates (tHM/yr), including initial cores and reloads;

- LWR and SFR UNF reprocessing rates (tIHM/yr);

- Unused TRU recovered from UNF (t), i.e., the remaining inventory (from both LWR and SFR UNF) after accounting for fuel fabrication needs in that year;

- $\quad$ LWR and SFR UNF in cooling storage $(t)$;

- LWR and SFR UNF waiting for reprocessing (after cooling storage) ( $\mathrm{t}$ ), i.e., the remaining inventory after the maximum amount of UNF is reprocessed in that year; this is 0 for SFR UNF since the capacity is unlimited.

Only annual values were requested, so the value types in the specifications had to address when these values are reported and whether rates are instantaneous or cumulative, as mentioned in the Methodology section. For example, all capacities and inventories are reported at the end of the year, after all of the reactors are started up or retired and all of the mass flows are accounted for. Note that a retiring reactor will generate its last electricity at the last time step of the year and then discharges its entire core at the first time step of the following year, as shown in Figure 2. All mass flow rates are cumulative for that year, e.g., the total amount (tIHM) of LWR UNF reprocessed in 2030.

First, the spreadsheet solutions are shown since the LWR retirement profile was calculated using this approach. Then, the results from each code are discussed, focusing on the main differences from the spreadsheet result. Attempting to match results from the fuel cycle codes to those from the spreadsheet posed some challenges because of fundamentally different model structures and assumptions. The spreadsheet solution is based on discrete changes (similar to delta functions or step changes) that occur simultaneously, whereas system dynamics codes assume continuous processes that occur with each time step with more realistic gradual changes. Matching the results exactly requires circumventing code features that were implemented to model the actual structure of the fuel cycle. Although this approach does not fully explore unique and important capabilities of the codes, it did expose key limitations and served to help build confidence and a better understanding of the core structure of the codes. Since this is simply a test scenario that was not optimized or claimed to be realistic, the results and their political, economic, and technological implications will not be discussed in detail. The focus is on matching the time-dependent values and trends as they apply to the verification of the different calculation approaches.

\subsection{Spreadsheet Results}

All four participating U.S. national laboratories used Excel to produce spreadsheet results for the test scenario based on the specifications. All were able to produce the same annual values as shown in Figures 4 through 10. The LWR/SFR fleet profile during transition, as previously shown in Table 4, is graphically shown in Figure 4. Figure 5 shows the corresponding LWR retirement and SFR start-up rates in terms of numbers of reactors (recall that each LWR is 3 times the electrical capacity of a single SFR). Notice that starting in 2086, the first wave of SFRs constructed between 2026 and 2055 start to retire and are replaced by new SFRs during this second wave.

Again, these retirement and start-up rates were calculated for a scenario trying to minimize the unused TRU inventory, as shown in Figure 6. Notice that the amount of unused TRU hovers very close to 0 until 2075, indicating that the system's reactor deployment strategy is indeed deploying the SFRs as soon as 
there is sufficient fuel. However, between 2055 and 2095, the LWR retirement rate (and effectively, the SFR start-up rate) is dramatically slowed down to build up enough TRU to fuel the second wave of SFR construction beginning in 2086. This leads to the significant spike in Figure 6 between 2075 and 2085. After the SFR fleet is fully deployed in 2096, the unused TRU inventory increases because the TRU inventory ratio of the SFR and fuel combination is 1.012. The additional $1.2 \%$ was designed to account for typical reprocessing and fabrication losses at $1 \%$ and $0.2 \%$, respectively. However, since no losses were assumed in this simulation, the buildup of TRU after 2096 was expected.

Figure 7 shows the annual fuel loading rates for both reactor types including first core and equilibrium reload fuel. These curves correspond to the deployed reactor capacities. Figure 8 shows the UNF discharged and stored on-site in cooling storage. After 4-year and 1-year cooling periods for the LWR and SFR fuels, respectively, they are moved to the reprocessing plant for immediate reprocessing if there is sufficient capacity. If reprocessing is not available yet or there is a backlog, the cooled UNF appears in Figure 9 as UNF waiting for reprocessing.

After the start of LWR UNF reprocessing in 2025, the LWR UNF reprocessing plant will be operating at its full capacity of 2,000 tIHM/yr during the first few decades, as shown in Figure 10. In 2055, there is no more LWR UNF in queue for reprocessing so the capacity factor of the LWR UNF reprocessing plant will suddenly drop since the small LWR fleet share at that time will discharge only a fraction of the 1,991 tons of UNF that it discharged in 2015. Therefore, the deployment rate of the SFRs must slow down due to the slower rate of TRU "production" from the LWRs. If the rapid SFR construction rate and corresponding LWR replacement rate were maintained after 2055, there will be insufficient TRU due to over-construction of SFRs (TRU sinks) and insufficient LWRs (TRU sources). This was among several important lessons learned through this artificial problem that was designed to push the system to the fastest transition by recycling as much fuel as possible at any time. In a more realistic fuel cycle transition scenario, there would be some margin added (e.g., storage buffers, slower SFR deployment, etc.) to account for uncertainty, timing and size differences, processing batches, etc.

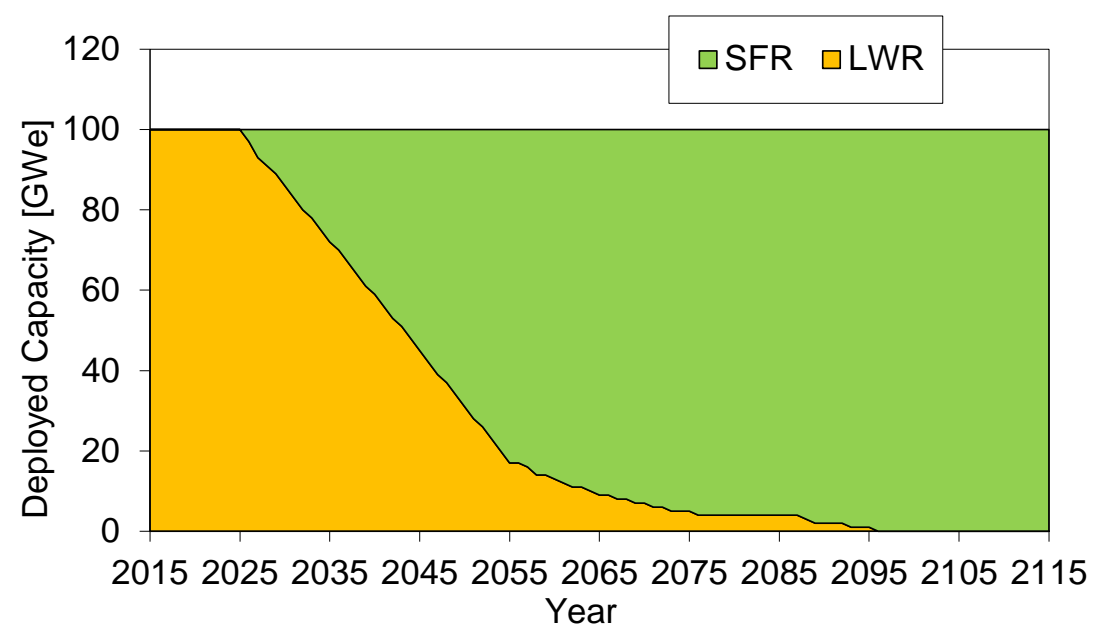

Figure 4 Deployed reactor capacities at the end of each year 


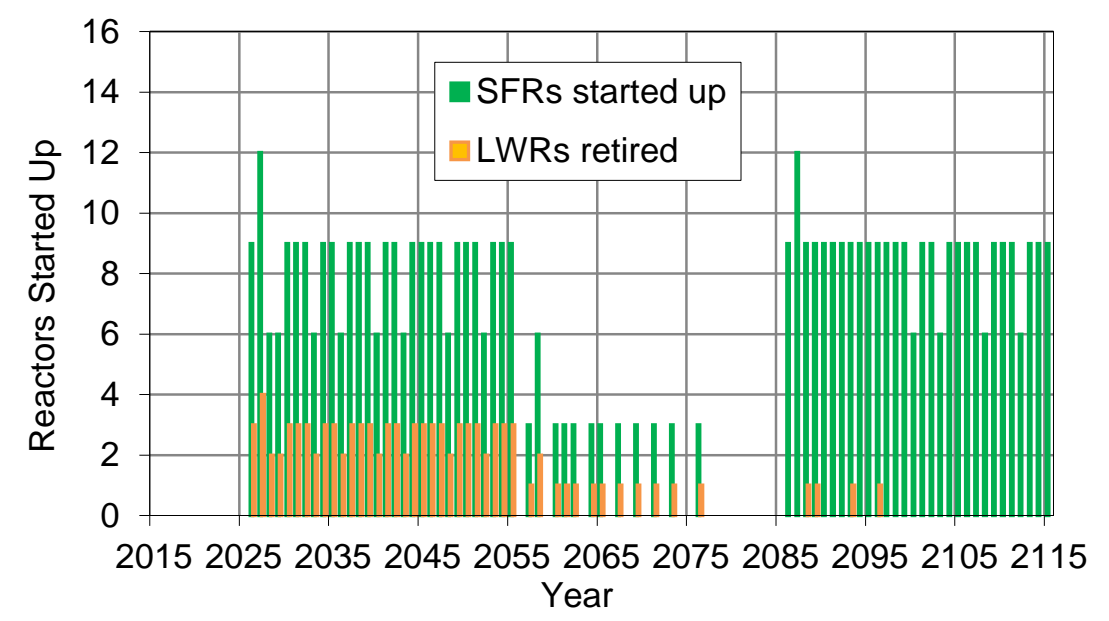

Figure 5 LWRs retired and SFRs started up each year

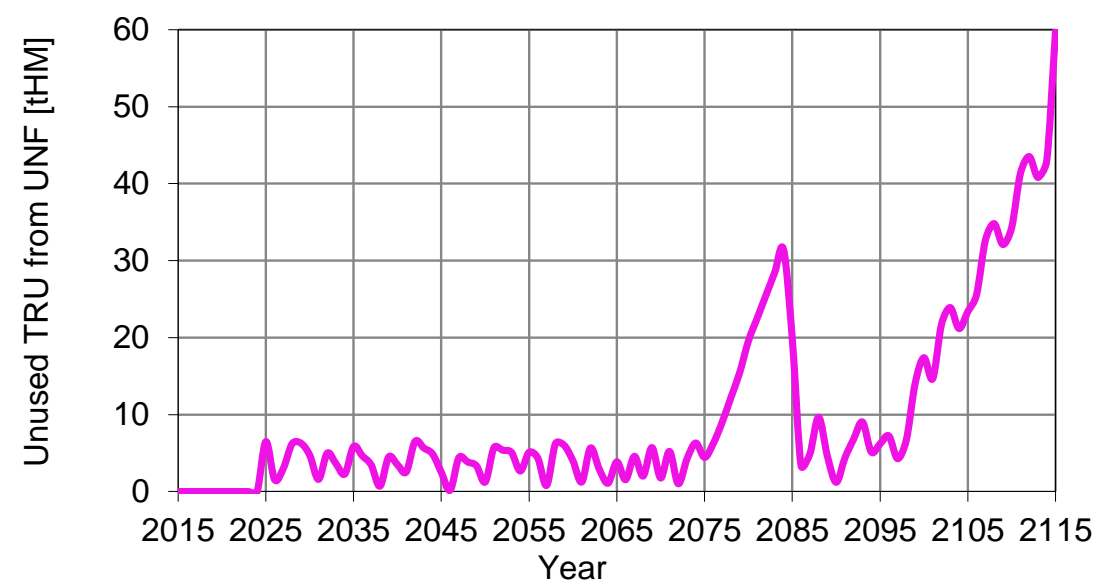

Figure 6 Inventory of unused TRU recovered from UNF (measured at end of year)

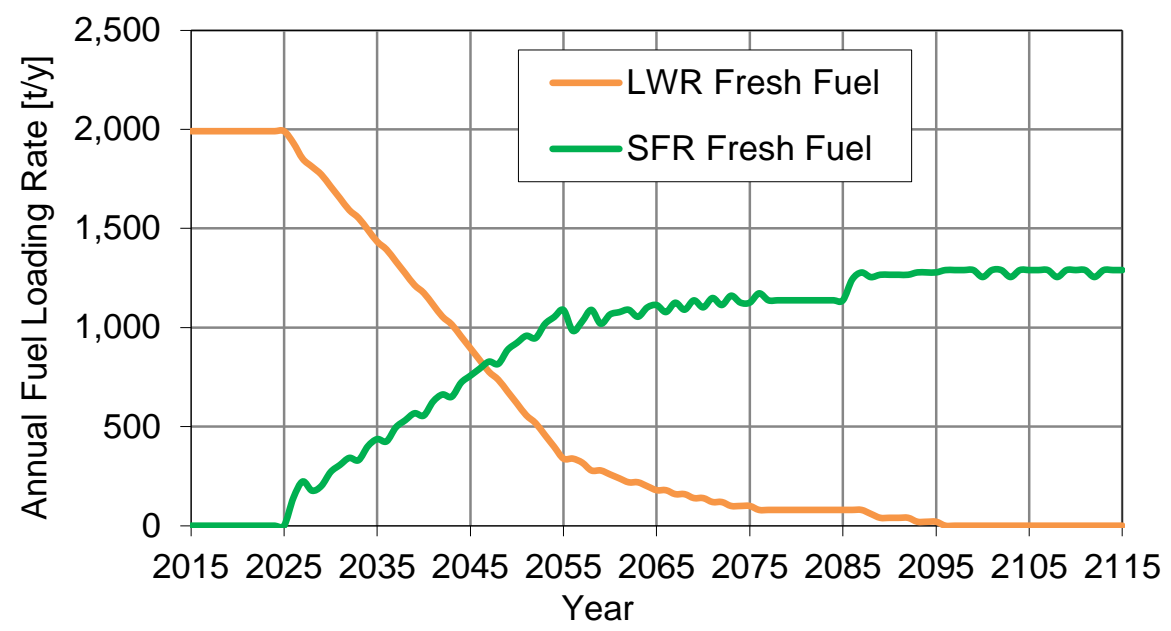

Figure 7 Annual fresh fuel loading rates (first cores and reload fuel) 


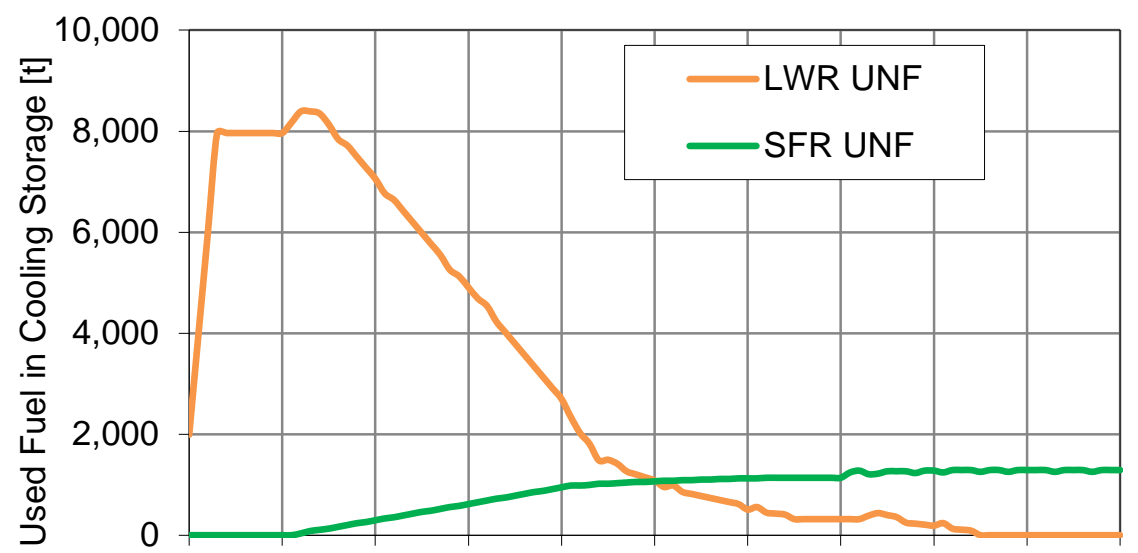

20152025203520452055206520752085209521052115

Year

Figure 8 Inventory of discharged UNF in mandatory cooling storage

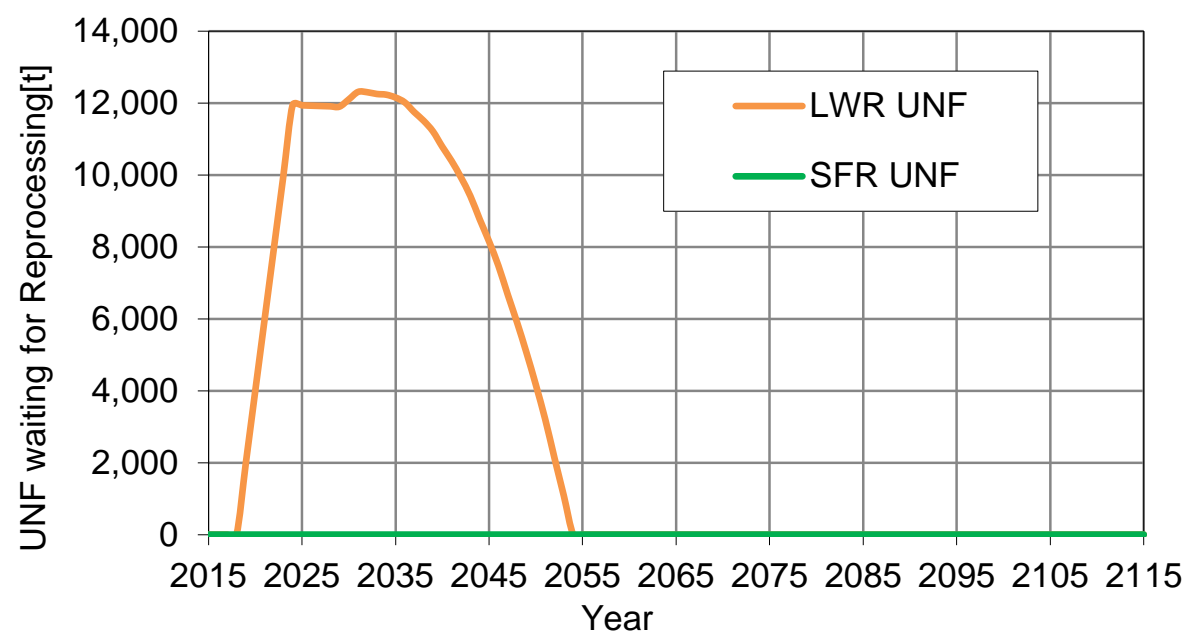

Figure 9 Inventory of discharged and cooled UNF waiting for reprocessing

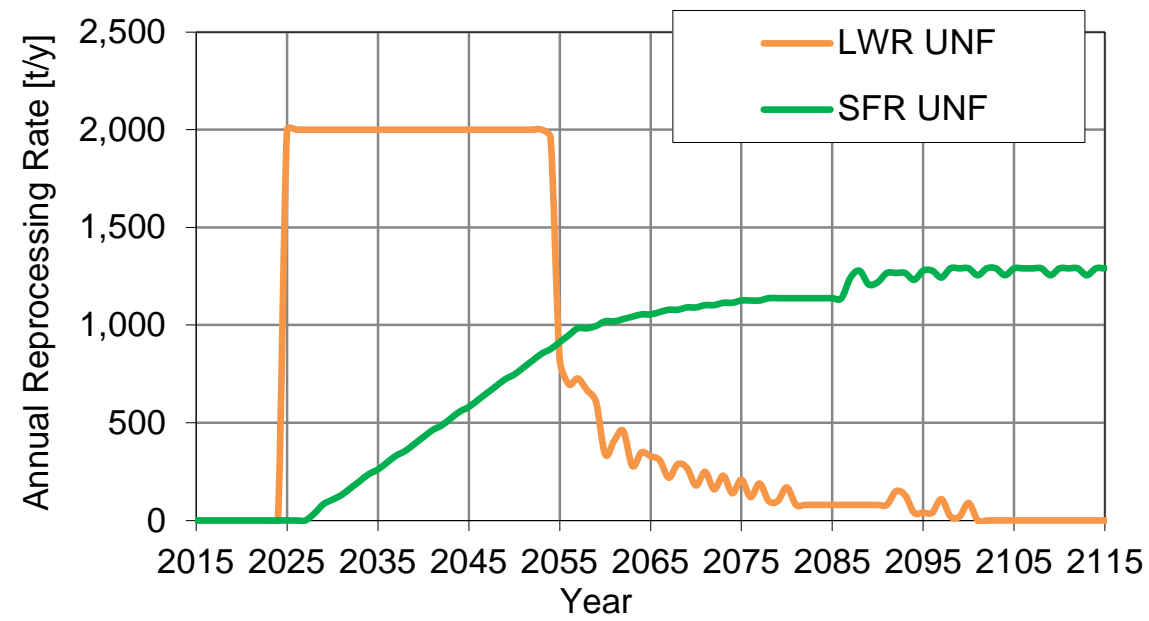

Figure 10 Annual reprocessing throughputs 


\subsection{DYMOND Results}

Like other codes involved in this study, DYMOND was slightly modified after finding a few code inconsistencies as a result of this verification exercise. The initial unmodified version of DYMOND produced results that agreed very well with the spreadsheet results; there was less than $4 \%$ difference (which was later reduced after code modifications) from the spreadsheet values averaged over the simulation period for all requested figures except for the unused TRU recovered from UNF, the indicator value that is typically one of the most difficult to match with different codes because it is a derivative quantity that is based on a variety of complex mass flows.

After numerous additional model runs, clarification of the inputs and assumptions, and cross-checking of results, the differences between the spreadsheet and DYMOND solutions were found to be mainly attributed to small differences on how first core loads were modeled. In general, DYMOND modeled refueling during the first cycle whereas the spreadsheet did not (which is more accurate). Therefore, modifications/corrections were made to the code that prevented refueling from taking place during the first cycle. This resulted in much closer agreement in the first cycle's mass flows with those of the spreadsheet. In addition, a few fixes were made to the time step synchronization from one process to another. Following the modifications, the final results for the indicator values specified in the code-tocode comparison produced exact matches (within numerical rounding error) for all plots, but large differences still remained for the unused TRU recovered from UNF, as shown in Figure 11 and those relating to SFR fuel mass flows. In addition, DYMOND's average annual SFR fuel mass flow values are between 1-2\% lower compared to the spreadsheet, which are difficult to discern visually in the figures, but still failed to meet the virtual match criterion met by the other calculated parameters. These apparent differences in the unused TRU and SFR mass flows are due to the difference in how reprocessing is modeled; the spreadsheets model a year's worth of reprocessing throughput instantaneously in the beginning of the year whereas DYMOND models reprocessing at each time step.

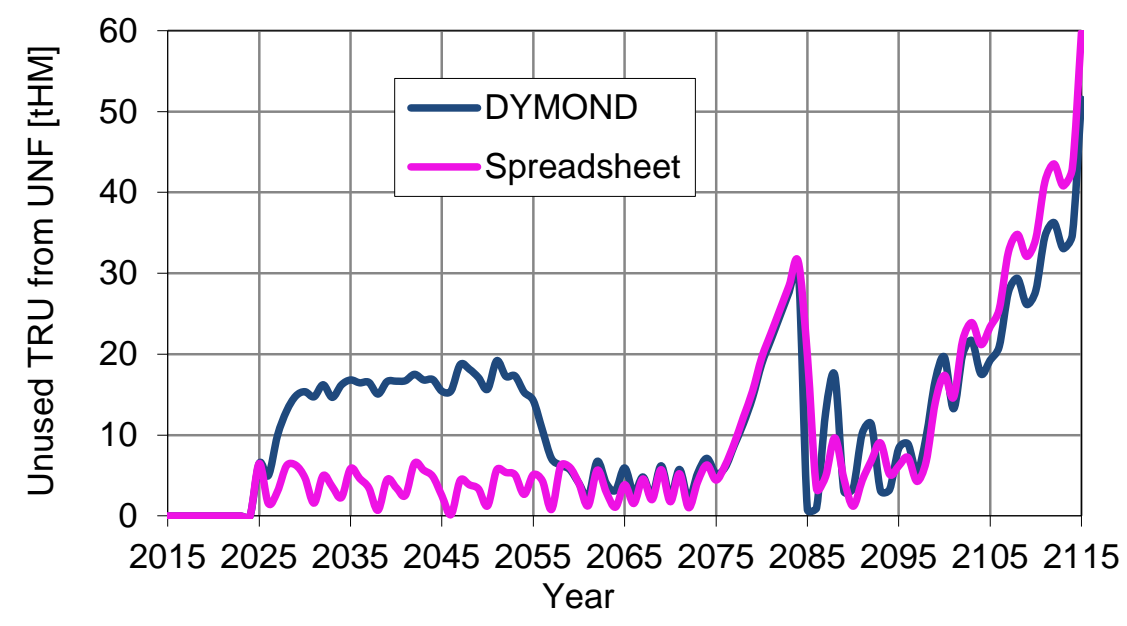

Figure 11 Unused TRU recovered from UNF calculated by DYMOND and spreadsheet

As mentioned in the specifications, the LWR reactor retirement profile shown in Table 4 and the associated SFR deployment profile were used as inputs into DYMOND and all other codes to help isolate the subtle differences in how mass flows were calculated. However, this reactor profile was calculated by the spreadsheets under the assumption that the annual reprocessing throughput of 2,000 tons of LWR UNF occurs instantly at the beginning of the year, which is an artifact of the coarse 1-year time steps used 
in the spreadsheet. This assumption would allow a year's worth of separated TRU from the reprocessed LWR UNF to be immediately available at the beginning of the year to fabricate fuel for the new SFR start-ups, which is a significant approximation. In contrast, DYMOND was set up to use $1 / 10$-year time steps and therefore spreads this 2000 tons/year of reprocessing capacity over the course of the year, which is more realistic with more refined time steps. Therefore, during the first time step of the year, the TRU from only 200 tons of reprocessed LWR UNF is available for SFR fuel fabrication in DYMOND. These two different reprocessing modeling approaches are shown in Figure 12.
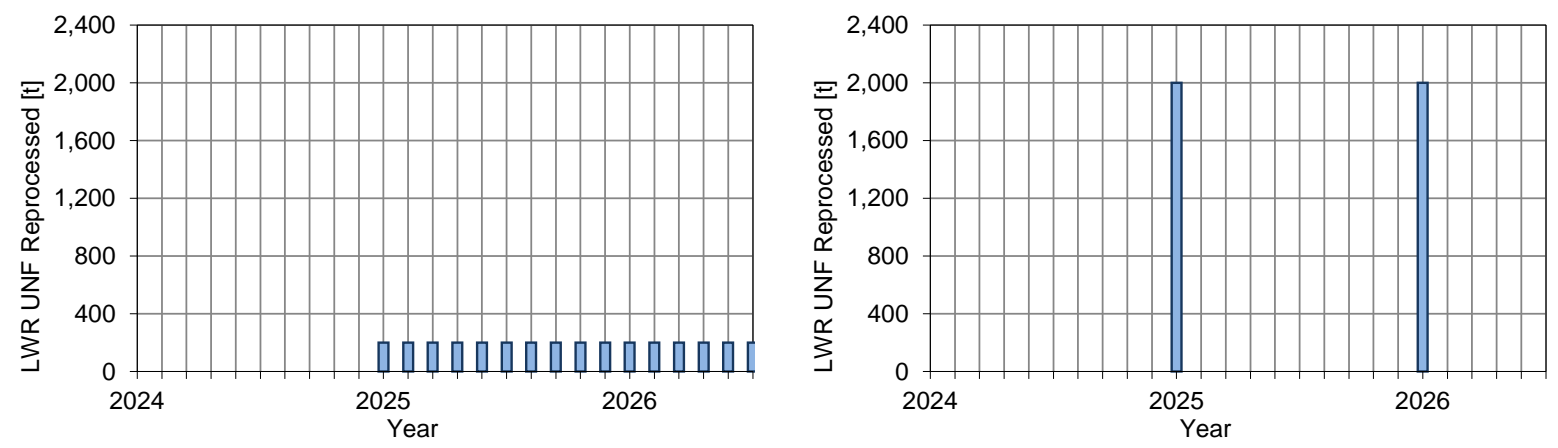

Figure 12 LWR used fuel reprocessing schedule modeled in DYMOND (L) and the spreadsheet (R)

This subtle difference in the LWR UNF reprocessing throughput "delays" the availability of the separated TRU for SFR fuel fabrication in DYMOND, resulting in a few time steps during which there is insufficient fuel for the deployed SFRs that have already been constructed according to the spreadsheetcalculated reactor profile. These unfueled SFRs are shown in Figure 13 as "idle capacities", which do not occur in the spreadsheet solution. Note that these are plotted per time-step, so the largest amount of idle capacity $(60,000 \mathrm{MWe})$ only occurs for $1 / 10$ of a year, not an entire year.

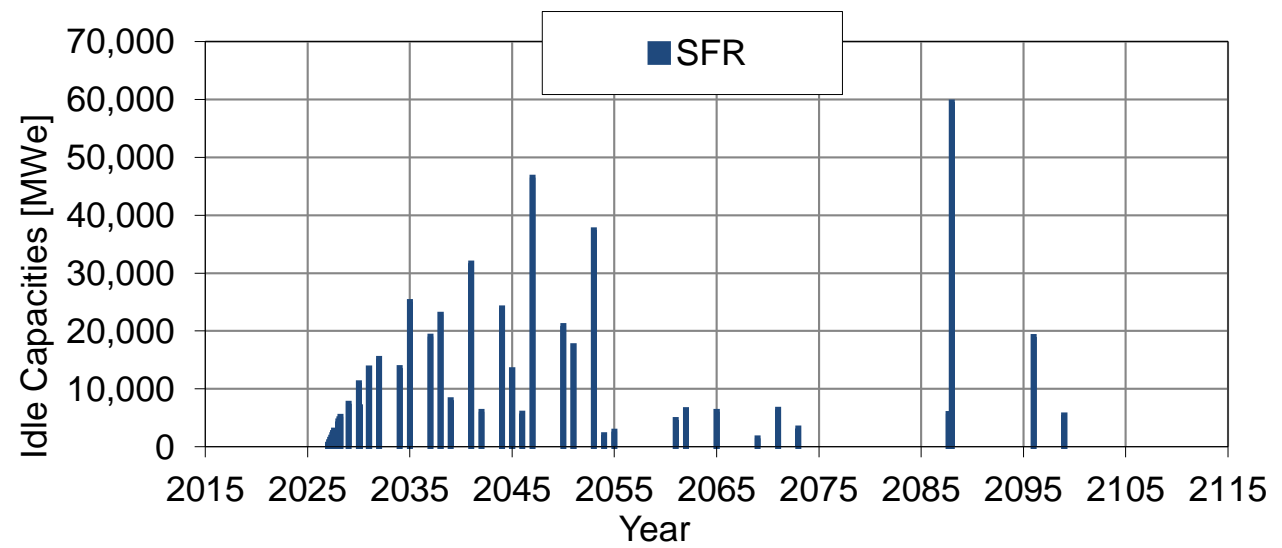

Figure 13 Capacity of unfueled reactors at each timestep calculated by DYMOND

The cumulative amount of idle capacities calculated in DYMOND is about 4.9 GWe-y of energy, which corresponds to about 600 tons of unloaded SFR fuel. This is consistent with the fact that DYMOND's cumulative throughput of SFR fuel $(89,055$ tHM loaded) over the 100 year period is 663 tons lower than that of the spreadsheets $(89,718$ tHM loaded). The remaining difference of less than 100 tons $(\sim 0.1 \%$ of 
total fuel loaded) can be largely attributed to rounding differences. This explains DYMOND's 1-2\% lower SFR-related annual mass flows compared to the spreadsheets.

In addition, this "late" arrival of separated TRU and resulting energy deficit of 4.9 GWe-y also explains DYMOND's greater unused TRU levels shown in Figure 11. The 600 tons of unloaded (or "late") SFR fuel corresponds to about 11 tons of unused TRU, which is essentially the difference in TRU levels between DYMOND and the spreadsheet between years 2025-2055. After 2055, this extra TRU is used to fabricate additional SFR fuel to temporarily reduce the amount of idle capacities until 2085, when the largest spike in idle capacity occurs. This spike is due to the replacement of the first-built SFRs after they have reached their assumed 60-year lifetime with new SFR start-ups. This results in extremely high SFR fuel requests during that first time step and as expected, insufficient SFR fuel available due to DYMOND's modeling of continuous instead of instant reprocessing. With the SFR fleet reaching its equilibrium state in 2096 (100\% SFRs) and a continually increasing surplus TRU inventory as shown in Figure 11, there should not be any additional idle capacity occurrences thereafter.

All of the differences in the results between DYMOND and the spreadsheets for this verification scenario can be directly attributed to the difference in how the reprocessing is modeled. This difference was not interpreted as a modeling inaccuracy in DYMOND, but rather as a large approximation made by the spreadsheet. Therefore, it was not deemed necessary to modify the code or change the assumptions to obtain closer agreement.

\subsection{VISION Results}

Similar to the DYMOND results, the VISION calculated values also showed excellent agreement with the spreadsheet solutions with the exception of the unused TRU recovered from UNF. In addition, there were a few minor differences related to the UNF inventories and reprocessing throughputs: As shown in Figure 14, after 2055, the VISION model showed a small, non-zero time-varying inventory of cooled LWR UNF, whereas the spreadsheet solution showed none. This is because VISION outputs not only the cooled LWR UNF waiting for reprocessing at each time step, but also the material which passes through to separations during the same time step. This is a consequence of the system dynamics modeling paradigm in use within the VISION code infrastructure. Specifically, the separated material requires at least one time step to transit through separated material storage on its way from separations to fuel fabrication and is reported as part of the inventory. The mean residency time within storage is expected to be quite short, except when a surplus of material occurs.

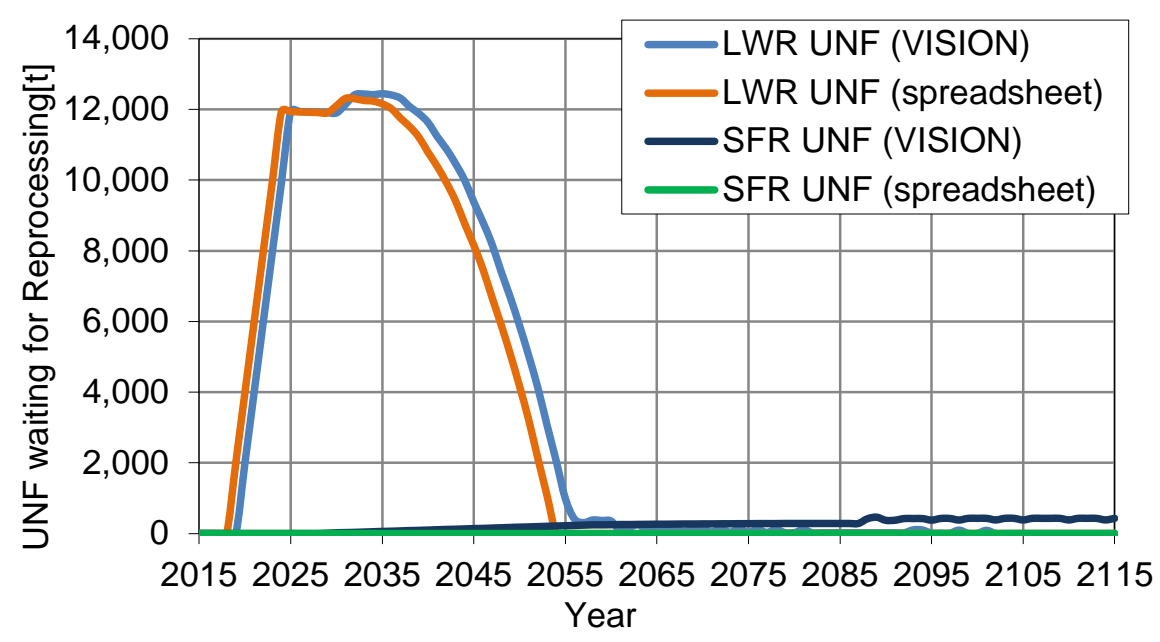

Figure 14 UNF waiting for reprocessing calculated by VISION and spreadsheet 
The UNF reprocessing rates were functionally identical between the VISION and spreadsheet solutions, as shown in Figure 15. There is a slight delay seen in the VISION results relative to the spreadsheet, which is an artifact of an offset in the VISION output and is not reflective of an underlying discrepancy in the model.

As mentioned, the only major difference between the VISION output and the spreadsheet output was the unused TRU recovered from UNF. As shown in Figure 16, the predicted inventory by VISION fluctuates at around 20 tons starting in 2025, which is closer to DYMOND's results than those of the spreadsheet. This is because VISION also models reprocessing at every time step instead of once per year. However, there is no sudden drop in the inventory between 2060 and 2075 like the DYMOND result. In addition, the characteristic sharp peak at around 2085 is higher by 20 tons compared to both DYMOND and the spreadsheet result. The higher inventory levels can also be attributed to how VISION reports the inventory in addition to the mass in transit for each time step.

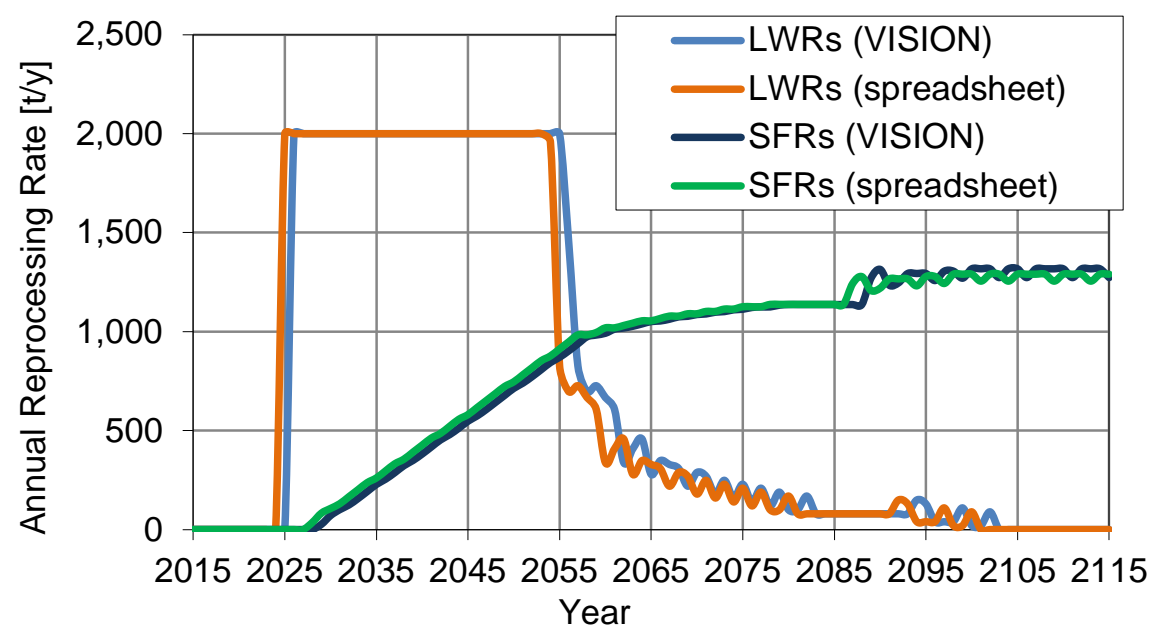

Figure 15 UNF reprocessing rate calculated by VISION and spreadsheet

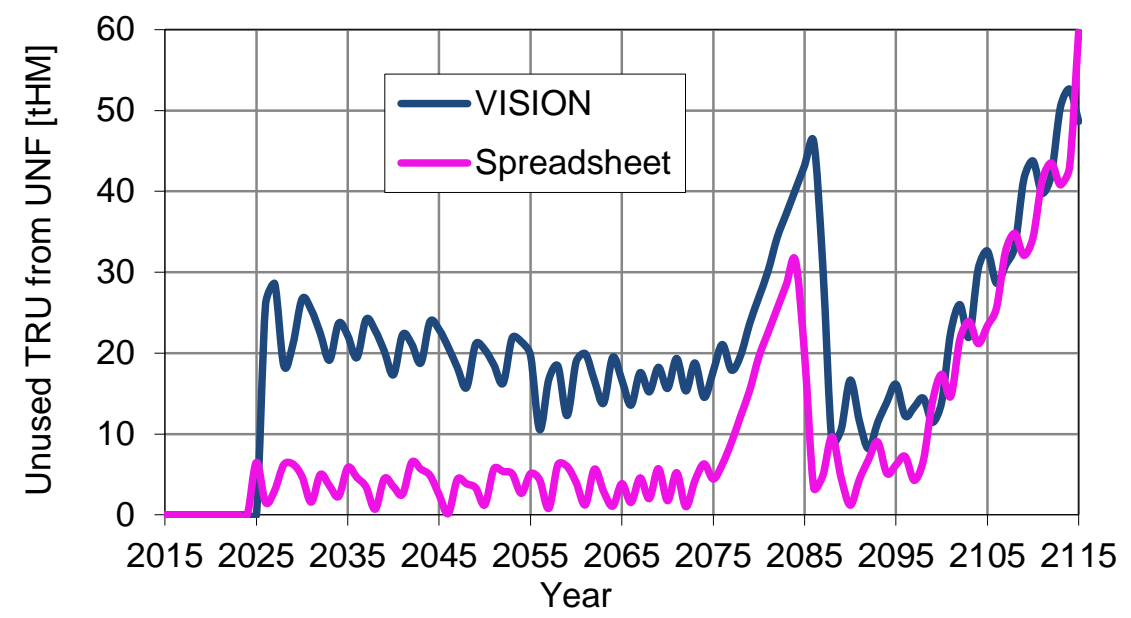

Figure 16 Unused TRU from UNF calculated by VISION and spreadsheet 
Several modifications to VISION were made as a result of this verification exercise. One key feature of the code is the ability to forecast the amount of material recovered from UNF available for use in recycling reactors. Figure 17 shows the number of reactors that are forecasted by VISION to experience fuel shortages in the future, which is an example application of this new forecasting feature to the verification scenario. This is a very informative plot because it closely resembles a corresponding figure produced by DYMOND (Figure 13). Although the units from Figures 13 and 17 are different, they are both similar in that the systems in VISION and DYMOND continuously incur fuel shortages or idle capacities from 2025 to 2055 , and then there are a few peaks shortly after 2085. These apparent fuel shortages are consistent with the lack of SFR fuel because of the aforementioned continuous reprocessing modeled in the two codes. There is ongoing work to reduce the difference between the forecast and actual fuel supply to extend the usability of the automatic ordering algorithm.

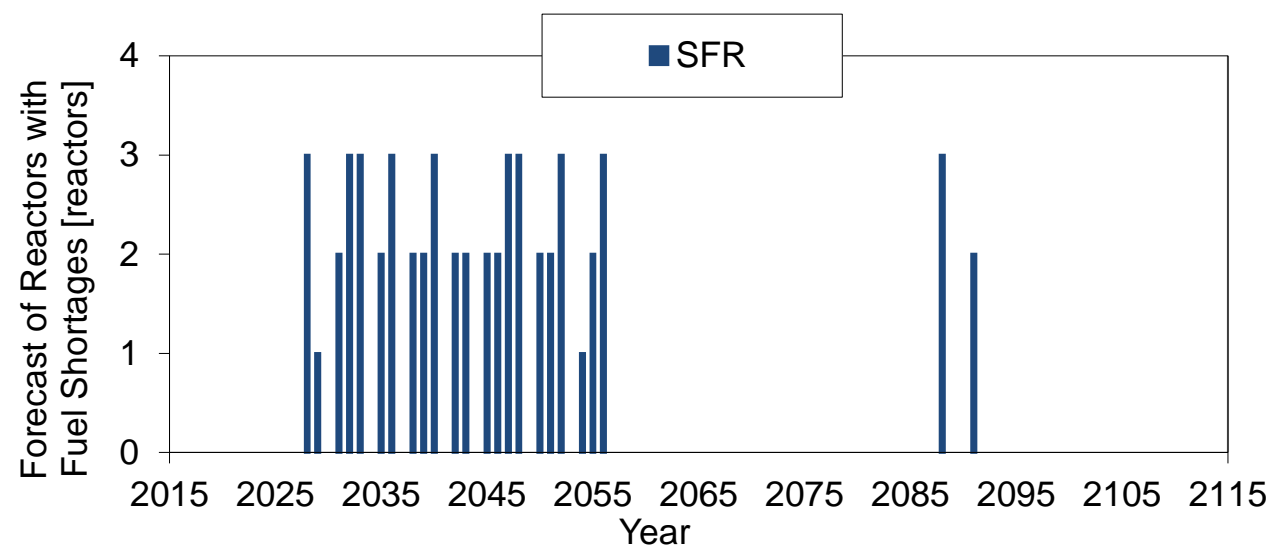

Figure 17 VISION-predicted number of reactors started up that will experience a fuel shortage at some point during operation

\subsection{ORION Results}

The ORION results showed excellent agreement with spreadsheet results for all identified indicators after accounting for differences related to the timesteps. Figure 18 shows the deployed SFR and LWR capacity results from ORION and the spreadsheet. The small differences are due to ORION automatically increasing the operational lifetimes of the LWR and SFR fleets such that they are divisible by the cycle length. ORION will also not decommission a reactor until the end of a fuel cycle has been reached, which also contributes to the apparent fluctuations in the LWR installed capacity profile, whereas in other codes, the commissioning or decommissioning of a reactor occurs at a finite timestep at the beginning or end of the year.

Figure 19 shows a comparison of ORION and spreadsheet results for the fresh fuel loading rates for both the LWR and SFR fleets. The ORION results for the LWR fuel loading rates appear to fluctuate because of the following reasons: 1) a time step and reporting interval of 6 months were used in ORION while the LWR cycle length is 18 months, 2) discrete batches of fuel were modeled instead of splitting an annual refueling mass flow over each time step, and 3) all 100 existing LWRs were modeled to start at the first time step of the simulation, so they are all fueled at the same time step. As a result, ORION outputs the instantaneous refueling rate instead of the annualized one from the spreadsheets and other codes. However, the year-averaged LWR fuel loading rates from ORION agree with the annual results obtained 
from the spreadsheet. For the SFR fuel loading rate, the ORION and the spreadsheet values in Figure 19 match because the SFR cycle length is exactly 1 year, so the instantaneous and year-averaged loading rates are the same. Similar behavior was also observed for other mass flow data such as the UNF reprocessing rates; fluctuations may appear due to the aforementioned reasons but the year-averaged results match those of the spreadsheet.

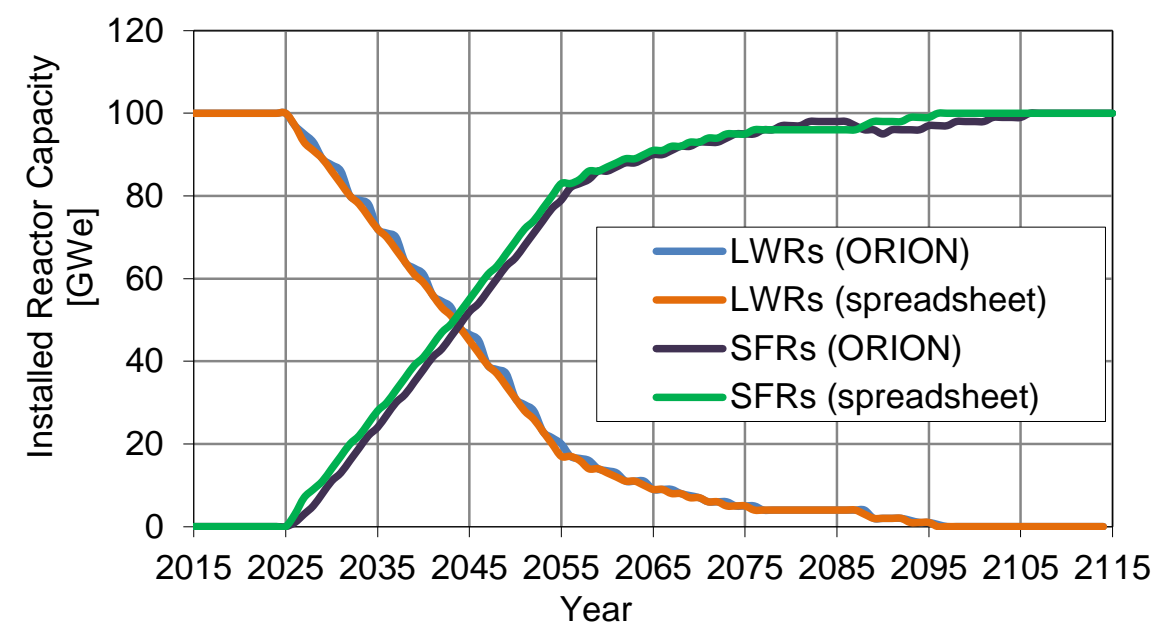

Figure 18 Installed reactor capacities from ORION and spreadsheet

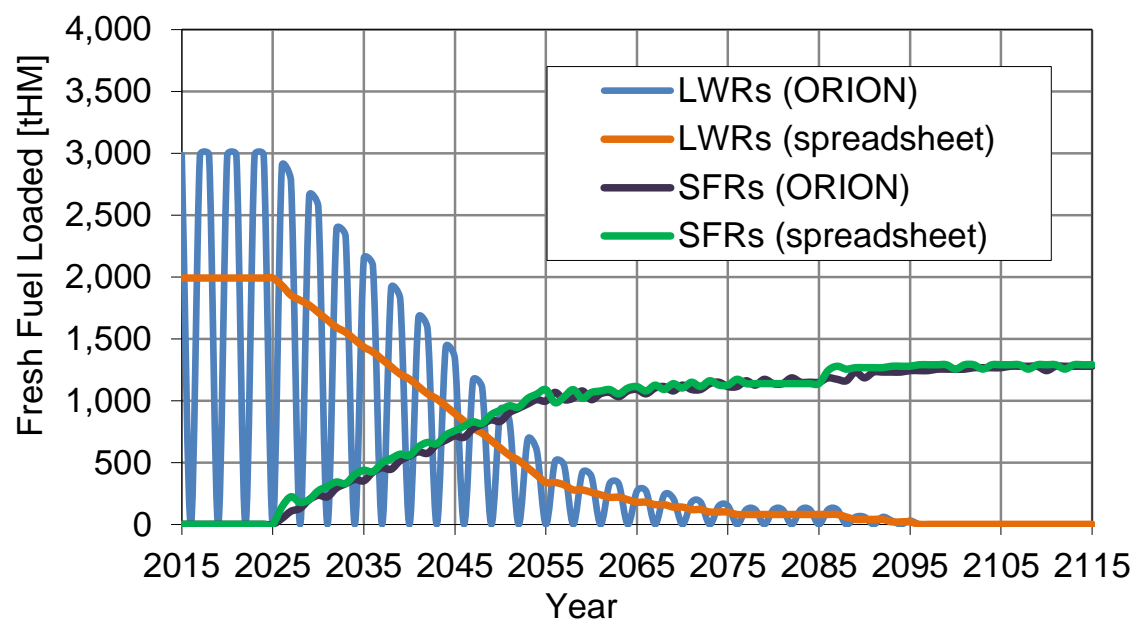

Figure 19 Fresh fuel loaded from ORION and spreadsheet

Figure 20 shows a comparison of the ORION and spreadsheet results for the cooled LWR fuel in storage waiting for reprocessing. Because only one value is reported for each year, it is vital to emphasize the exact reporting time for each of the inventory curves. Two lines for the spreadsheet results, one before and one after the allocated amount of fuel is transferred to reprocessing for each year, are shown in Figure 20 to emphasize this aspect and to help explain the differences with the ORION result. The ORION results are reported every 6 months, so the cooled LWR fuel inventory fluctuates between the two spreadsheet-produced lines that are reported every 12 months. From inspection, one can see that if year- 
averaged fuel inventories were shown from both the ORION and spreadsheet, then they would match. Similar differences were observed for the LWR fuel in cooling storage results.

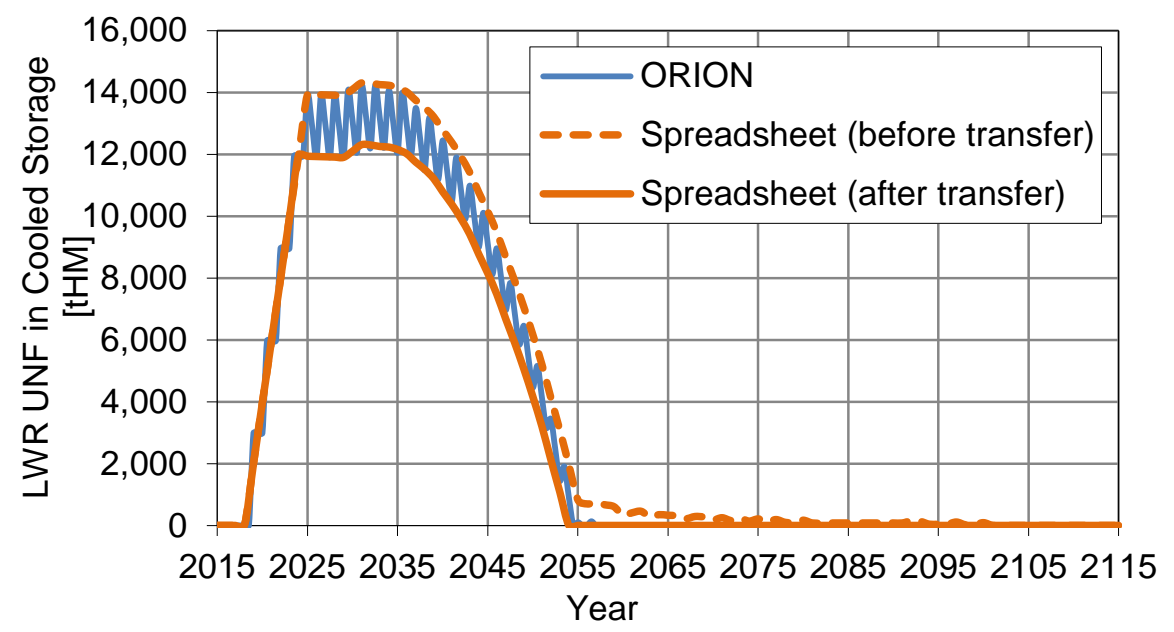

Figure 20 Comparison of ORION and spreadsheet results for LWR UNF in cooled storage

\subsection{MARKAL Results}

For this verification exercise, a standalone MARKAL model (i.e., not embedded in the U.S. energy system model already within the code) was developed, as shown as a schematic mass flow model in Figure 21. Each box represents a separate "technology" in MARKAL. Each technology has an associated input and output material and/or energy form. The black boxes are process technologies and the red boxes are stockpile technologies. The stockpile technologies are used to: a) model an unlimited supply of UOX; or b) accumulate the material generated in the previous step (process). All process technologies have been modeled with a lag, i.e., output materials exit the process one time step after the corresponding input materials enter it. The processes labeled "LAG" carry an input material to the next time step with no other change, and represent the cooling period after the spent nuclear fuel is discharged from a reactor. The activity of the "Reprocess LWR UNF" technology was assigned an upper bound of $2000 \mathrm{t} / \mathrm{y}$ after 2025. The MARKAL results were obtained using the exact LWR retirement profile (and SFR start-up profile) described in Table 4. The main difficulty faced in this verification exercise stemmed from the fact that MARKAL only allows for 60 time-steps, and two consecutive runs were necessary to simulate the entire time period under consideration. The data from the first run (2015-2075) were manually carried into the second calculation. Similar to the other codes, MARKAL's model had to be modified several times to ensure consistency with the specifications; initially some of the results (e.g., "Unused TRU recovered from UNF") lagged behind the spreadsheet solution because MARKAL reported outputs at different time points (e.g., after fabrication instead of after reprocessing).

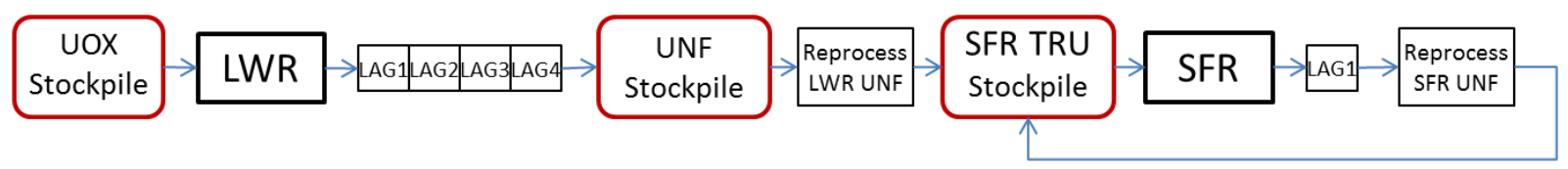

Figure 21 Mass flow schematic of the MARKAL model for the test scenario 
Since the MARKAL model used a pre-defined retirement profile and used 1 year time steps like the spreadsheet, all MARKAL-generated data matched exactly with those from the spreadsheet except for the unused TRU from UNF, as shown in Figure 22. Visually, it appears that the two lines are matched at all times, but the third line indicates that a small difference (within 3\%) appears after 2075, which is simply due to the difference in numerical precision of the simulation data that is transferred between the two 60year MARKAL simulations. Otherwise, all of the MARKAL and spreadsheet results matched at all times for this test scenario.

The verification of MARKAL under this test transition scenario demonstrated the ability for the code to accurately model evolving nuclear fuel cycle systems. The standalone model developed for this verification exercise was subsequently integrated into a model of the entire U.S. energy system including nuclear and non-nuclear energy sources. This comprehensive energy model is being used to examine the future deployment rates of nuclear energy in the U.S as it competes with other sources under various assumptions and conditions (carbon limits, electricity prices, etc.). The resulting information, such as the projected growth rates for nuclear power, can then be used as boundary conditions for future nuclear transition analyses.

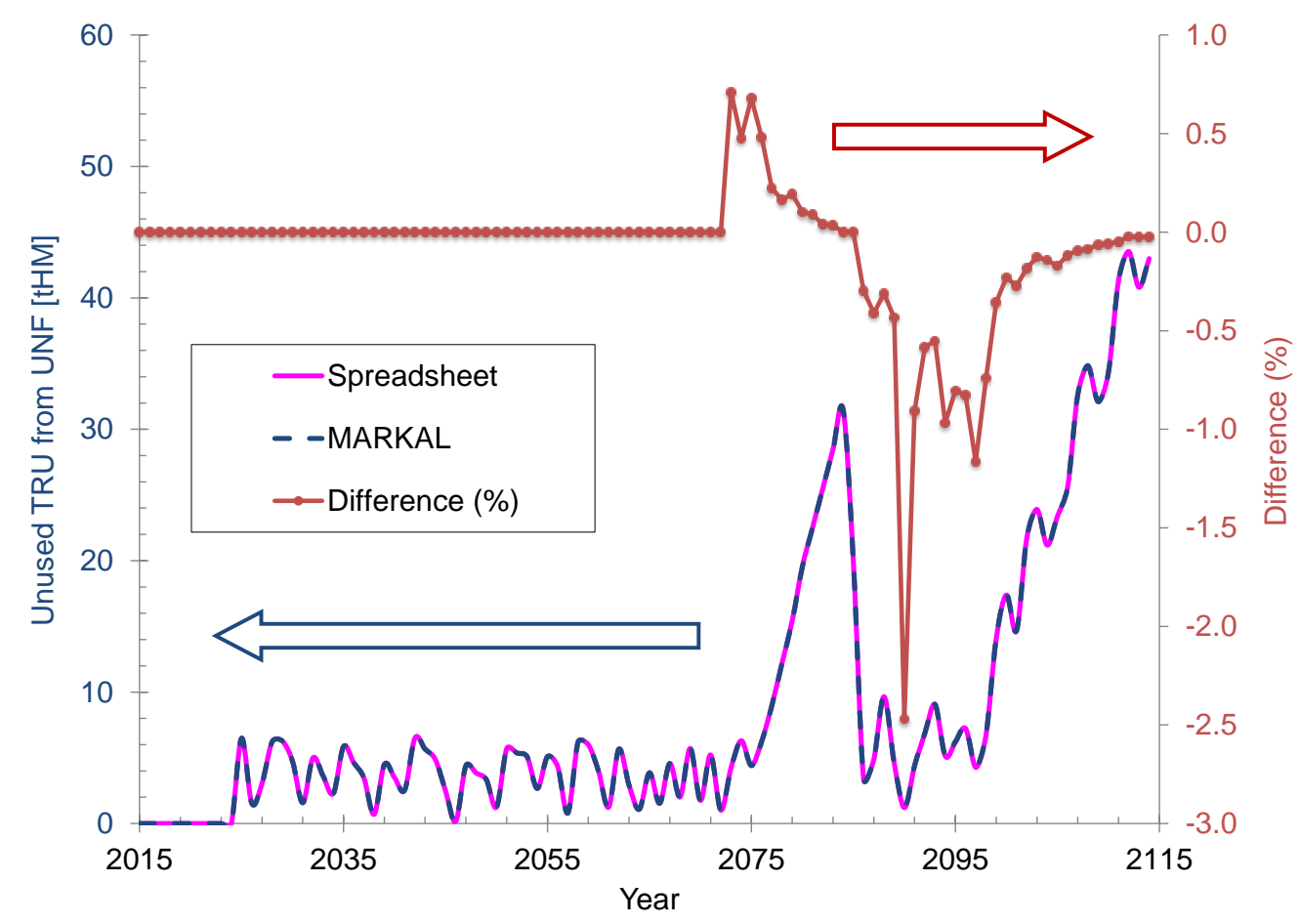

Figure 22 Unused TRU recovered from UNF from MARKAL and spreadsheet with the percent difference indicated on the right 


\section{Conclusions}

Using a unified set of benchmark specifications and problem interpretations, excellent agreement for the test scenario results was achieved between all four codes and the spreadsheet, although each had a few remaining differences due to fundamental differences in code structure and functions. For example, DYMOND and VISION model continuous reprocessing at every time step instead of instantaneous reprocessing (as simulated in the spreadsheet), so there is a "delay" in the recovery of fissile materials to be used in recycled fuel. ORION explicitly models the timing of each fuel reload in between cycle lengths instead of averaging the mass flow over each time step (like in DYMOND and VISION) or over a year (as was done in the spreadsheet calculation). This feature in conjunction with other modeling assumptions (synchronized start-up of all initial LWRs) and display differences (cycle-averaged reporting instead of year-averaged), resulted in the apparent "oscillations" in the ORION results. Under different assumptions, modeling individual batches may be more realistic, but with a large fleet of reactors, the averaging of mass flows over each time step should result in very small differences. MARKAL was able to match the spreadsheet results the closest largely because the MARKAL model adopted the same reactor profile and year-long time steps for all processes and outputs.

The use of the spreadsheet was an effort to confirm simple arithmetic that is often hidden in the complex infrastructure of the codes so as to not treat them as "black boxes". During the early stages of this effort, each participant independently developed their own spreadsheet model and produced identical results. All of these results had a common accounting error that was revealed after using one of the codes, indicating that spreadsheets, like codes, are also prone to human errors in development and interpretation. In addition, simple spreadsheet models have their own limitations that sophisticated fuel cycle codes can overcome: the time steps for the calculation are not easily adjusted, simulating a large number of isotopes becomes cumbersome, modeling isotopic decay and determining time-dependent properties such as radioactivity are difficult, etc. For the analysis of new fuel cycles, an entirely new spreadsheet model is likely to be needed, rather than adapting the existing tools, as is the case with a dedicated fuel cycle simulator.

It should be emphasized again that a majority of the initial differences between the code and spreadsheet results were due to different interpretations of the specifications among the analysts, therefore a single interpretation for each input and output had to be established among all the participants. This required frequent updates to the test scenario specifications with less ambiguous language. Through such a rigorous process in which codes and modeling specifications are iteratively corrected or modified to remove any unexplainable differences, this study was able to not only verify four fuel cycle codes for a test transition scenario, but also demonstrate an effective quality control approach. By upholding multiple code results to such a high level of agreement and scrutiny, stakeholders and decision makers can have more confidence in future results generated via this process that can help inform on potential strategic and policy decisions. In addition to this iterative verification approach, the test scenario specifications and results can also be used to involve new or additional codes for future verification studies. However, it must be recognized that the presented test scenario is an artificial problem in which the last bit of material is recycled as soon as possible to support the transition rate. In addition, the reference results were calculated using the spreadsheet approach with 1-year time steps that was later revealed by the code results to be essential for this transition to work. A more realistic scenario with some added margin and flexibility (such as adding fuel storage buffers and/or more gradual SFR deployment rates) would help account for uncertainties and differences attributed to the different time steps. These results would likely

not include any idle capacities or insufficient fuel, thereby allowing smoother behavior and closer agreement. 
Although the codes were able to be verified for the test scenario, additional calibration and minor modifications may still be needed for future scenarios that may differ in terms of growth rate, number of reactor technologies, different fuel cycle processes, etc. At the very least, due to the potential for large differences in results with large implications, it is recommended to use a diverse toolset in a manner that reduces the uncertainty and increases the level of confidence in the results. Finally, all fuel cycle simulations are only models and not real systems, so any such "model-induced" behaviors need to be identified, explained, and minimized so as to reduce the potential for misinterpretation of results by decision makers.

\section{Acknowledgements}

This work was funded through the Fuel Cycle Options Campaign within the U.S. DOE Office of Nuclear Energy (Fuel Cycle Technologies). The work performed by Argonne National Laboratory is supported under U.S. Department of Energy contract DE-AC02-06CH11357.

This paper has greatly benefited from the insight and teamwork from all contributors to the Fuel Cycle Options Campaign spanning across many national laboratories, universities, and industry, to which the authors are very grateful. It also serves as an excellent example of the product resulting from an open, collaborative team environment that can be established across multiple institutions and code developers.

\section{References}

MIT 2009

EC-PUMA 2010

NEA 2012

Yacout et al. 2005

Jacobson et al. 2010
Guérin, L., B. Feng, P. Hejzlar, B. Forget, M.S. Kazimi, L. Van Den Durpel, A. Yacout, T. Taiwo, B. Dixon, G. Matthern, L. Boucher, M. Delpech, R. Girieud, and M. Meyer, "A Benchmark Study of Computer Codes for System Analysis of the Nuclear Fuel Cycle,” MIT-NFC-105, April (2009).

Kuijper, J.C. et al., "Plutonium and Minor Actinide Management in Thermal High-Temperature Gas-Cooled Reactors - Publishable Final Activity Report," Technical Report, p. 74-79, European Commission - Euratom Research and Training Programme on Nuclear Energy, June (2010).

OECD/NEA, "Benchmark Study on Nuclear Fuel Cycle Transition Scenarios Analysis Codes," NEA/NSC/WPFC/DOC(2012)16, June (2012).

Yacout, A.M., J. J. Jacobson, G. E. Matthern, S. J. Piet, and A. Moisseytsev, "Modeling the Nuclear Fuel Cycle," The 23rd International Conference of the System Dynamics Society, Boston, MA, July 17-21 (2005).

Jacobson, J.J., A. M. Yacout, G. E. Matthern, S. J. Piet, D. E. Shropshire, R. F. Jeffers, and T. Schweitzer, "Verifiable Fuel Cycle Simulation Model (VISION): A Tool for Analyzing Nuclear Fuel Cycle Futures," Nuclear Technology, 172, 157-178, November (2010). 
Dixon et al. 2010 Dixon B., B. Halsey, S. Kim, G. Mathern, S. Piet, and D. Shropshire, "Dynamic Systems Analysis Report for Nuclear Fuel Recycle," Advanced Fuel Cycle Initiative, INL/EXT-08-15201, December (2008).

Gregg and Grove 2012 Gregg, R. and C. Grove, "Analysis of the UK nuclear fission roadmap using the ORION fuel cycle modeling code," Proceedings of the IChemE Nuclear Fuel Cycle Conference, Manchester, United Kingdom (2012).

Shay et al. 2006

Shay, C., J. DeCarolis, D. Loughlin, C. Gage, S. Yeh, S. Vijay, and E. Wright, "EPA U.S. National MARKAL Database: Database Documentation", U.S. Environmental Protection Agency Report EPA-600/R-06/057, February (2006).

Wigeland et al. 2014 Wigeland, R., T. Taiwo, H. Ludewig, M. Todosow, W. Halsey, J. Gehin, R. Jubin, J. Buelt, S. Stockinger, K. Jenni, and B. Oakley, "Nuclear Fuel Cycle Evaluation and Screening - Final Report," FCRD-FCO-2014-000106, Fuel Cycle Technologies Program, October 8 (2014). 